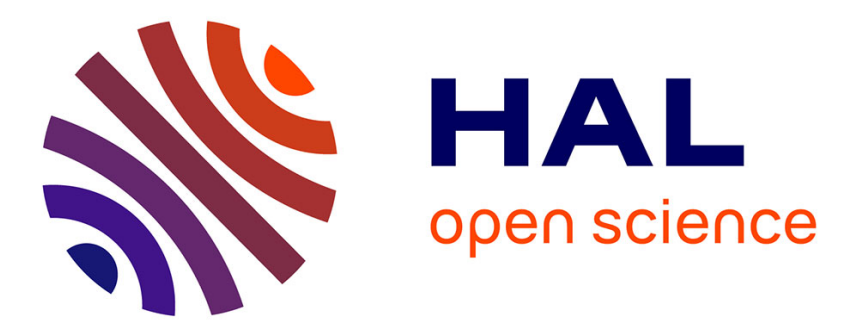

\title{
Improving convergence in numerical analysis using observers - The wave-like equation case
}

Dominique Chapelle, Nicolae Cîndea, Philippe Moireau

\section{To cite this version:}

Dominique Chapelle, Nicolae Cîndea, Philippe Moireau. Improving convergence in numerical analysis using observers - The wave-like equation case. Mathematical Models and Methods in Applied Sciences, 2012, 22 (12), 10.1142/S0218202512500406 . inria-00621052v2

\section{HAL Id: inria-00621052 \\ https://hal.inria.fr/inria-00621052v2}

Submitted on 1 Mar 2012

HAL is a multi-disciplinary open access archive for the deposit and dissemination of scientific research documents, whether they are published or not. The documents may come from teaching and research institutions in France or abroad, or from public or private research centers.
L'archive ouverte pluridisciplinaire HAL, est destinée au dépôt et à la diffusion de documents scientifiques de niveau recherche, publiés ou non, émanant des établissements d'enseignement et de recherche français ou étrangers, des laboratoires publics ou privés. 


\title{
March $\quad 2012 \quad 13: 17$ WSPC/INSTRUCTION FILE waveSDFNumAnal-m3as
}

Mathematical Models and Methods in Applied Sciences

(c) World Scientific Publishing Company

\section{Improving convergence in numerical analysis using observers The wave-like equation case}

\author{
D. Chapelle \\ Inria, Rocquencourt, B.P. 105 \\ 78153 Le Chesnay, France \\ dominique.chapelle@inria.fr \\ N. Cîndea * \\ Inria, Rocquencourt, B.P. 105 \\ 78153 Le Chesnay, France \\ nicolae.cindea@inria.fr \\ P. Moireau \\ Inria, Rocquencourt, B.P. 105 \\ 78153 Le Chesnay, France \\ philippe.moireau@inria.fr \\ Received (11 Sept. 2011) \\ Revised (10 Febr. 2012) \\ Accepted (28 Febr. 2012) \\ Communicated by (xxxxxxxxxx)
}

\begin{abstract}
We propose an observer-based approach to circumvent the issue of unbounded approximation errors - with respect to the length of the time window considered - in the discretization of wave-like equations in bounded domains, which covers the cases of the wave equation per se and of linear elasticity as well as beam, plate and shell formulations, and so on. Namely, taking advantage of some measurements available on the system over time, we adopt a strategy inspired from sequential data assimilation and by which the discrete system is dynamically corrected using the discrepancy between the solution and the measurements. In addition to the classical cornerstones of numerical analysis made up by stability and consistency, we are thus led to incorporating a third crucial requirement pertaining to observability - to be preserved through discretization. The latter property warrants exponential stability for the corrected dynamics, hence provides bounded approximation errors over time. Special care is needed to establish the required observability at the discrete level, in particular due to the fact that we focus on an original observer method adapted to measurements of the main variable, whereas measurements of the time-derivative - admissible, of course, albeit less frequent in practical systems - lead to a stability analysis in which existing results can be more directly applied. We also provide some detailed application examples with several such wave-like
\end{abstract}

* Current address: Laboratoire de Mathématiques, UMR CNRS 6620, Campus des Cézeaux, BP 80026, 63171, Aubière, France, Nicolae.Cindea@math.univ-bpclermont.fr 
equations, and the corresponding numerical assessments illustrate the performance of our approach.

Keywords: data assimilation; Luenberger observers; numerical analysis with measurements; stabilization of conservative systems.

AMS Subject Classification: 35L05, 35L20, 65M12, 93B07, 93B40, 93D15

\section{Introduction}

The numerical analysis of evolution partial differential equations (PDEs) is a wellestablished field, in particular as regards second-order problems in the time variable - see e.g. Refs. 9, 35 - such as "wave-like" equations in bounded domains, which covers the cases of the wave equation per se and of linear elasticity as well as beam, plate and shell formulations, and so on. In this context, it is well-known that, not only the error estimates derived in numerical analysis, but also the actual errors observed in numerical experiments frequently suffer from a steady deterioration over time - even when only spatial semi-discretization is considered - rendering the numerical solution virtually useless beyond a rather limited time range. Concurrently, it should be noted that these classical discretization procedures heavily rely on the accurate knowledge of the initial condition - a rather strong assumption - as the only type of information at hand on the solution. However, in many instances of actual systems a more reasonable assumption is instead that a limited accuracy prevails on the initial condition, albeit some measurements are also available on the solution of interest at subsequent times, e.g. measured values in a specific subset of the domain with a given time sampling. The key idea in this paper is to take advantage of such measurements in order to circumvent the above difficulty of unbounded error estimates over time.

To that purpose, we adopt an observer approach, namely, we incorporate in the system a consistent perturbation based on the discrepancy between the solution and the measurements. This type of methodology, introduced in the 60's in the control community with the pioneering "Luenberger observers" 24 - and later developed somewhat independently in engineering under the "nudging" terminology - was primarily devised in the framework of state estimation to cope with uncertain initial conditions, and relies on the idea that a well-chosen such perturbation may stabilize the system dynamics. As a consequence the estimation error which follows the stabilized dynamics can effectively converge to zero. These methods were initially proposed for systems of rather limited sizes, and indeed only much more recently have they been considered for infinite-dimensional systems modeled by PDEs - as such in Refs. 39, 18, 15, 34 and under the impetus of data assimilation in Refs. 28, 1 - as an effective alternative to sequential methods based on optimization principles - in particular the Kalman filter or various methods derived thereof - which are not easily suited to such systems due to the "curse of dimensionality" coined by R.E. Bellman.

In our case, we also aim at exploiting the stabilization principle underlying 
the concept of observers, albeit here in order to tackle consistency errors arising from the discretization rather than - or in addition to - initial condition errors usually considered in data assimilation. Therefore we will undertake the complete numerical analysis of the "closed-loop" observer system for wave-like equations and show that - under some natural observability conditions - we can obtain error estimates that no longer deteriorate with the simulation duration, thereby providing a dramatic improvement over direct discretizations of the original system. As our strategy will crucially rely on the analysis of the discrete error equations - for which the continuous counterpart is a stabilized equation as already mentioned - we will be able to benefit from earlier works on the numerical analysis of stabilized systems and the preservation of uniform stability at the discrete level. ${ }^{10,13}$ Indeed, to the classical cornerstones of numerical analysis formed by stability and consistency, we add an observability condition associated with the data employed and required to ensure the enhanced stability in the observer, including at the discrete level. However, stabilization terms considered in earlier works are mainly based on time-derivatives of the equation unknown - i.e. velocity-like quantities - hence the corresponding observers require measuring the same type of quantities. Here, we rather focus on direct (although partial) measurements of the unknown itself, in a context where time-differentiating the data should be avoided because of measurement errors which is frequently the case in practice - and our preferred observer method is the so-called "Schur Displacement Feedback" proposed in Ref. 29, see also Ref. 22 for germane ideas. The resulting error equation is then rather non-standard and necessitates some further analysis. We point out that observer strategies and their discretizations can also be employed for other purposes - beyond sequential state estimation - not further discussed in this article, such as parameter estimation, ${ }^{28,6,27}$ or reconstruction of the initial condition. ${ }^{34,16}$

The outline of the paper is as follows. In Section 2 we introduce the problem setting and state our main error estimation result (Th. 2.1). Sec. 3 is then devoted to establishing the required discrete observability property, while in Sec. 4 we complete the proof of Th. 2.1 by deriving and analyzing the discrete system obeyed by the approximation error. Next, in Sec. 5 we demonstrate the potential of our approach on application examples concerning the wave equation, with numerical assessments given in space dimensions 1 and 2 , before turning our attention to beam and plate models in Sec. 6.

\section{Problem setting and main result}

Let $\mathcal{H}$ be a Hilbert space of inner product and associated norm denoted by $\langle\cdot, \cdot\rangle$ and $\|\cdot\|$, respectively. Let $A_{0}: \mathcal{D}\left(A_{0}\right) \rightarrow \mathcal{H}$ be a self-adjoint, definite positive operator with compact resolvent. We consider the following system

$$
\left\{\begin{array}{l}
\ddot{w}(t)+A_{0} w(t)=0, \quad t>0 \\
w(0)=w_{0}, \quad \dot{w}(0)=w_{1}
\end{array}\right.
$$


which we refer to as a "wave-like system". Let $\mathcal{Z}$ be another Hilbert space and let $H_{0} \in \mathcal{L}\left(\mathcal{D}\left(A_{0}^{\frac{1}{2}}\right), \mathcal{Z}\right)$ defining the following output function

$$
z(t)=H_{0} w(t)
$$

Definition 2.1. System (2.1)-(2.2) is exactly observable in time $T>0$ if there exists $k_{T}>0$ such that any solution of $(2.1)$ for $\left(w_{0}, w_{1}\right) \in \mathcal{D}\left(A_{0}\right) \times \mathcal{D}\left(A_{0}^{\frac{1}{2}}\right)$ satisfies

$$
\int_{0}^{T}\left\|H_{0} w(t)\right\|_{\mathcal{Z}}^{2} \mathrm{~d} t \geq k_{T}\left(\left\|A_{0}^{\frac{1}{2}} w_{0}\right\|^{2}+\left\|w_{1}\right\|^{2}\right) .
$$

System (2.1)-(2.2) is said to be exactly observable if there exists any $T>0$ so that (2.1)-(2.2) is exactly observable in time $T$.

Denoting $x(t)=\left(\begin{array}{c}w(t) \\ w(t)\end{array}\right)$, we can rewrite (2.1) as the first-order system

$$
\left\{\begin{array}{l}
\dot{x}(t)=A x(t), \quad t>0 \\
x(0)=x_{0}
\end{array}\right.
$$

where $A: \mathcal{D}(A) \rightarrow \mathcal{X}$ with

$$
\mathcal{D}(A)=\mathcal{D}\left(A_{0}\right) \times \mathcal{D}\left(A_{0}^{\frac{1}{2}}\right), \quad \mathcal{X}=\mathcal{D}\left(A_{0}^{\frac{1}{2}}\right) \times \mathcal{H}, \quad A=\left(\begin{array}{cc}
0 & I \\
-A_{0} & 0
\end{array}\right),
$$

and $x_{0}=\left(\begin{array}{c}w_{0} \\ w_{1}\end{array}\right)$. Moreover, we define $H \in \mathcal{L}(\mathcal{X}, \mathcal{Z})$ by

$$
H=\left(H_{0} 0\right),
$$

and $H$ is called the observation operator.

The essential aim of this paper is to show that when discretizing (2.1) we can obtain better convergence properties if we use the measurements of the system state corresponding to (2.2), via an adequate observer methodology. In order to state this result precisely we now introduce some definitions and notation. Assume that there exists a family $\left(\mathcal{V}_{h}\right)_{h>0}$ of finite dimensional subspaces of $\mathcal{D}\left(A_{0}^{\frac{1}{2}}\right)$ such that for some $\theta \geq 1$ and $h^{*}>0$, we have for every $h \in\left(0, h^{*}\right)$

$$
\begin{gathered}
\left\|\varphi_{h}\right\|_{\mathcal{D}\left(A_{0}^{\frac{1}{2}}\right)} \leq C h^{-1}\left\|\varphi_{h}\right\|, \quad \forall \varphi_{h} \in \mathcal{V}_{h}, \\
\left\|\pi_{h} \varphi\right\|_{\mathcal{D}\left(A_{0}^{\frac{1}{2}}\right)} \leq C\|\varphi\|_{\mathcal{D}\left(A_{0}^{\frac{1}{2}}\right)}, \quad \forall \varphi \in \mathcal{D}\left(A_{0}^{\frac{1}{2}}\right), \\
\left\|\pi_{h} \varphi-\varphi\right\| \leq C h^{\theta}\|\varphi\|_{\mathcal{D}\left(A_{0}^{\frac{1}{2}}\right)}, \quad \forall \varphi \in \mathcal{D}\left(A_{0}^{\frac{1}{2}}\right), \\
\left\|\pi_{h} \varphi-\varphi\right\|_{\mathcal{D}\left(A_{0}^{\frac{1}{2}}\right)} \leq C h^{\theta}\|\varphi\|_{\mathcal{D}\left(A_{0}\right)}, \quad \forall \varphi \in \mathcal{D}\left(A_{0}\right), \\
\left\|\check{\pi}_{h} \varphi-\varphi\right\| \leq C h^{\theta}\|\varphi\|_{\mathcal{D}\left(A_{0}^{\frac{1}{2}}\right)}, \quad \forall \varphi \in \mathcal{D}\left(A_{0}^{\frac{1}{2}}\right),
\end{gathered}
$$




$$
\left\|\check{\pi}_{h} \varphi-\varphi\right\|_{\mathcal{D}\left(A_{0}^{\frac{1}{2}}\right)} \leq C h^{\theta}\|\varphi\|_{\mathcal{D}\left(A_{0}\right)}, \quad \forall \varphi \in \mathcal{D}\left(A_{0}\right),
$$

where $\pi_{h}$ and $\check{\pi}_{h}$ are the orthogonal projectors onto $\mathcal{V}_{h}$ with respect to the inner products of $\mathcal{H}$ and $\mathcal{D}\left(A_{0}^{\frac{1}{2}}\right)$, respectively, and with $C$ denoting a generic strictly positive constant independent of $h$. Note that (2.6) is a standard inverse inequality, while (2.8) and (2.11) represent usual interpolation properties of projection operators for finite element spaces. As regards (2.7) and (2.9), they would typically be established by using the inverse inequality (2.6), whereas (2.10) would be proven by a duality argument "à la Aubin-Nitsche" assuming some regularity on the domain. Now, defining $A_{0 h} \in \mathcal{L}\left(\mathcal{V}_{h}\right)$ by

$$
\left\langle A_{0 h} \varphi_{h}, \psi_{h}\right\rangle=\left\langle A_{0}^{\frac{1}{2}} \varphi_{h}, A_{0}^{\frac{1}{2}} \psi_{h}\right\rangle, \quad \forall \varphi_{h}, \psi_{h} \in \mathcal{V}_{h},
$$

we consider $0<\lambda_{1}^{h} \leq \lambda_{2}^{h} \leq \cdots \leq \lambda_{n_{h}}^{h}$ the eigenvalues of $A_{0 h}$, with associated normalized eigenvectors $\left(\phi_{j}^{h}\right)_{j=1, \cdots, n_{h}}$, and we can define

$$
\mathcal{C}_{h}(\eta)=\operatorname{span}\left\{\phi_{j}^{h} \text { such that } \lambda_{j}^{h} \leq \eta\right\} .
$$

We also introduce the operator $H_{0 h} \in \mathcal{L}\left(\mathcal{V}_{h}, \mathcal{Z}\right)$ given by the restriction of $H_{0}$ to $\mathcal{V}_{h}$, and we define $\mathcal{Z}_{h}=\operatorname{Im} H_{0 h}$. By construction, the adjoint operator $H_{0 h}^{*}$ is in $\mathcal{L}\left(\mathcal{Z}, \mathcal{V}_{h}\right)$.

In order to construct a first-order discrete system, we introduce $\mathcal{X}_{h}=\mathcal{V}_{h} \times \mathcal{V}_{h}$ endowed with the inner product of $\mathcal{X}$ - namely, $\mathcal{D}\left(A_{0}^{\frac{1}{2}}\right) \times \mathcal{H}$ - and we define $A_{h} \in$ $\mathcal{L}\left(\mathcal{X}_{h}\right)$ and $H_{h} \in \mathcal{L}\left(\mathcal{X}_{h}, \mathcal{Z}\right)$ given by

$$
A_{h}=\left(\begin{array}{cc}
0 & I \\
-A_{0 h} & 0
\end{array}\right), \quad H_{h}=\left(\begin{array}{ll}
H_{0 h} & 0
\end{array}\right) .
$$

In addition we define the combined projector

$$
\Pi_{h}: \mathcal{X} \rightarrow \mathcal{X}_{h}, \quad \Pi_{h}\left(\begin{array}{c}
\varphi \\
\psi
\end{array}\right)=\left(\begin{array}{c}
\check{\pi}_{h} \varphi \\
\pi_{h} \psi
\end{array}\right) .
$$

We can now consider the full discretization of (2.4) using the following midpoint time discretization

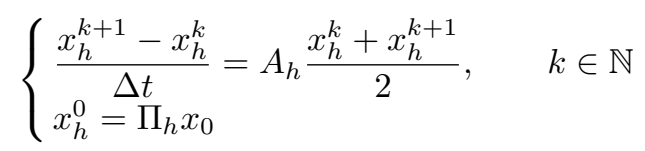

where $x_{h}^{k}$ is meant to approximate $x(k \Delta t)$. The numerical analysis of this totally discrete scheme is classical, e.g. using discrete Gronwall inequalities or Duhamel's formula combined with the conservative property of the discrete operators - we also refer to Ref. 35 for an analysis based on spectral decomposition. Under adequate regularity assumptions on the initial conditions and the domain, these estimates are of the type

$$
\left\|x_{h}^{n}-x(n \Delta t)\right\|_{\mathcal{X}} \leq C(T)\left(h^{\theta}+\Delta t^{2}\right)
$$


where the constant $C(T)$ indicates a dependence - typically linear - on the total simulation time $T=n \Delta t$, namely, this estimate is not bounded with respect to $T$.

By contrast, the main objective - and result - of the present paper is to show that, using some available data on the system (2.4), we can propose a fully discrete numerical scheme which approximates (2.4) with an error controlled by the discretization parameters (namely, $h$ and $\Delta t$ ) uniformly over time, i.e. without any dependence on the total simulation time.

To that purpose the key idea is to construct a discrete scheme inspired from the so-called observer system defined by

$$
\left\{\begin{array}{l}
\dot{\hat{x}}(t)=A \hat{x}(t)+H^{*}(z-H \hat{x}(t)), \quad t>0 \\
\hat{x}(0)=\hat{x}_{0}
\end{array}\right.
$$

Such an observer is designed to "track" the reference system, starting from a different initial condition, and we have the following dynamics for the observation error $\tilde{x}=x-\hat{x}$

$$
\left\{\begin{array}{l}
\dot{\tilde{x}}(t)=\left(A-H^{*} H\right) \tilde{x}(t), \quad t>0 \\
\tilde{x}(0)=x_{0}-\hat{x}_{0}
\end{array}\right.
$$

It is well-known that the exact observability property $(2.3)$ is equivalent to the asymptotic (exponential) stability of this error system - see e.g. Refs. 17, 23 and references therein - hence to the exponential convergence of the observer towards the reference. Noting that $\left(A-H^{*} H\right)$ also represents the governing operator in the dynamics of $\hat{x}$, we shall then proceed to discretize the observer system in place of the reference system, in essence to benefit from the enhanced stability of $\left(A-H^{*} H\right)$ compared to $A$. This in fact will lead to discrete error systems with accordingly modified dynamics, albeit non-homogeneous - unlike (2.16) - due to consistency error terms. In addition, in practice these discrete error systems should also incorporate measurement errors which may influence the accuracy of the proposed method depending on the specific measurement technology considered. Here, we do not want to dwell on the details of measurement error modeling, so we disregard these terms for simplicity, but they are further discussed in Remark 4.3 below, see also Ref. 28 and Ref. 6 for probabilistic and deterministic treatments, respectively.

Remark 2.1 (Stabilized dynamics). With the observation operator applying on the first part of the state variable as assumed in (2.5), denoting by $\hat{x}=\left(\begin{array}{c}\hat{w} \\ \hat{u}\end{array}\right)$ the observer tracking $x=\left(\begin{array}{c}w \\ \dot{w}\end{array}\right)$, the first-order observer dynamics equation can be decomposed into

$$
\left\{\begin{array}{l}
\dot{\hat{w}}=\hat{u}+H_{0}^{*}\left(z-H_{0} \hat{w}\right) \\
\dot{\hat{u}}+A_{0} \hat{w}=0
\end{array}\right.
$$

namely, the observer changes the canonical equation relating $w$ and $\dot{w}$, and likewise for the error equation (2.16), see Ref. 30. Therefore, while of course in this case " $H^{*} H$ " cannot be used to stabilize a physical system, it is perfectly admissible 
for an observer system - synthetic by construction, in the sense that it is always eventually numerically simulated - and leads to original closed-loop dynamics.

However, as discussed in particular in Ref. 13 (for a different observation operator) some special care must be exercised in order to preserve the exponential stability of such stabilized dynamics operators, since standard discretizations - in time and space - in general do not retain this property. As this difficulty is due to the (numerical) high frequency poles for which the real part is not adequately controlled, we can handle this as in Ref. 13 - see also Refs. 37, 33 for similar ideas - by incorporating a small perturbation based on a so-called "viscosity operator" $V_{\varepsilon} \in \mathcal{L}\left(\mathcal{X}_{h}\right)$, assumed to be self-adjoint and definite negative, and satisfying the additional requirements:

(1) Defining the orthogonal projector $\Pi_{h}^{\varepsilon}$ from $\mathcal{X}_{h}$ onto $\left(\mathcal{C}_{h}(1 / \varepsilon)\right)^{2}$ we have the commuting property

$$
\Pi_{h}^{\varepsilon} V_{\varepsilon}=V_{\varepsilon} \Pi_{h}^{\varepsilon}
$$

(2) There exist $c>0$ and $C>0$ such that

$$
\left\{\begin{array}{lc}
\sqrt{\varepsilon}\left\|\left(-V_{\varepsilon}\right)^{\frac{1}{2}} y\right\|_{\mathcal{X}} \leq C\|y\|_{\mathcal{X}}, & \forall y \in\left(\mathcal{C}_{h}(1 / \varepsilon)\right)^{2} \\
\sqrt{\varepsilon}\left\|\left(-V_{\varepsilon}\right)^{\frac{1}{2}} y\right\|_{\mathcal{X}} \geq c\|y\|_{\mathcal{X}}, & \forall y \in\left(\mathcal{C}_{h}(1 / \varepsilon)^{\perp}\right)^{2}
\end{array}\right.
$$

(3) We have the continuity estimate

$$
\begin{aligned}
\left\|V_{\varepsilon} \Pi_{h}\left(\begin{array}{c}
\varphi \\
\psi
\end{array}\right)\right\|_{\mathcal{X}} \leq C \varepsilon\left(\|\varphi\|_{\mathcal{D}\left(A_{0}^{\frac{3}{2}}\right)}+\|\psi\|_{\mathcal{D}\left(A_{0}\right)}\right), & \\
& \forall(\varphi, \psi) \in \mathcal{D}\left(A_{0}^{\frac{3}{2}}\right) \times \mathcal{D}\left(A_{0}\right) .
\end{aligned}
$$

With this viscosity operator and the data collected on the reference system assumed to be in the form

$$
z^{k}=H x(k \Delta t)
$$

we can finally define the following modified discretized system

$$
\left\{\begin{array}{l}
\frac{\widetilde{\bar{x}}_{h}^{k+1}-\bar{x}_{h}^{k}}{\Delta t}=A_{h} \frac{\bar{x}_{h}^{k}+\widetilde{\bar{x}}_{h}^{k+1}}{2}+\gamma H_{h}^{*}\left(\frac{z^{k}+z^{k+1}}{2}-H_{h} \frac{\bar{x}_{h}^{k}+\widetilde{\bar{x}}_{h}^{k+1}}{2}\right) \\
\frac{\bar{x}_{h}^{k+1}-\widetilde{\bar{x}}_{h}^{k+1}}{\Delta t}=\varepsilon V_{\varepsilon} \bar{x}_{h}^{k+1} \\
\bar{x}_{h}^{0}=\Pi_{h} x_{0},
\end{array}\right.
$$

where $\gamma>0$ is a fixed parameter. As stated in the following theorem, with this discretization scheme we can establish uniform-in-time error estimates, which shows that the above-mentioned objective is achieved.

Theorem 2.1. Let $A_{0}: \mathcal{D}\left(A_{0}\right) \rightarrow \mathcal{H}$ be a self-adjoint, definite positive operator with compact resolvent and let $H_{0} \in \mathcal{L}\left(\mathcal{D}\left(A_{0}^{\frac{1}{2}}\right), \mathcal{Z}\right)$ be a bounded linear operator such 
that System (2.1)-(2.2) is exactly observable. Let $\left(\mathcal{V}_{h}\right)_{0<h<h^{*}}$ be a family of finite dimensional subspaces of $\mathcal{D}\left(A_{0}^{\frac{1}{2}}\right)$ such that (2.6)-(2.11) hold, and let $\Delta t \in\left(0, \Delta t^{*}\right)$ be the time discretization step. Set $\varepsilon=\max \left\{\Delta t, h^{\theta}\right\}$ and let $V_{\varepsilon} \in \mathcal{L}\left(\mathcal{X}_{h}\right)$ be a viscosity operator satisfying the properties (2.17)-(2.19).

Then, considering the discrete system (2.20), for an initial condition $x_{0} \in$ $\mathcal{D}\left(A_{0}^{\frac{3}{2}}\right) \times \mathcal{D}\left(A_{0}\right)$ there exists a positive constant $C\left(x_{0}\right)$, independent of $h, \Delta t$ and $n$, such that

$$
\left\|\bar{x}_{h}^{n}-x(n \Delta t)\right\|_{\mathcal{X}} \leq C\left(x_{0}\right) \max \left(\varepsilon, \varepsilon^{2} h^{-1} \Delta t\right), \quad \forall n \in \mathbb{N} .
$$

Remark 2.2 (Observation versus stabilization). We point out that we formally use in observation the exact counterpart of the discretization strategy proposed in Th. 3.7 of Ref. 13 for stabilization purposes. However, in observation we need to deal with additional consistency issues due to the fact that the reference solution does not vanish, which is why we introduce the new condition (2.19). Moreover, Th. 3.7 of Ref. 13 assumes a discrete observability condition, established for various cases in Ref. 10 based on the corresponding continuous observability condition. Here, we only assume a continuous observability condition, for a class of observation operators which does not fall within the assumptions of Ref. 10.

Note that one natural - very straightforward for implementation purposes, indeed - example of viscosity operator is given by the following extension of the so-called "Rayleigh damping",

$$
V_{\varepsilon}=\varepsilon\left(A_{h}\right)^{2}=\varepsilon\left(\begin{array}{cc}
-A_{0 h} & 0 \\
0 & -A_{0 h}
\end{array}\right)
$$

This operator satisfies by construction the requirements $(2.17)-(2.18)$ with $c=C=$ 1 , and we can verify $(2.19)$ with

$$
\left\|V_{\varepsilon} \Pi_{h}\left(\begin{array}{c}
\varphi \\
\psi
\end{array}\right)\right\|_{\mathcal{X}}^{2}=\left\|A_{0 h} \check{\pi}_{h} \varphi\right\|_{\mathcal{D}\left(A_{0}^{\frac{1}{2}}\right)}^{2}+\left\|A_{0 h} \pi_{h} \psi\right\|^{2},
$$

and

$$
\begin{aligned}
\left\|A_{0 h} \pi_{h} \psi\right\| & =\sup _{\psi_{h} \in \mathcal{V}_{h}} \frac{\left\langle A_{0 h} \pi_{h} \psi, \psi_{h}\right\rangle}{\left\|\psi_{h}\right\|}=\sup _{\psi_{h} \in \mathcal{V}_{h}} \frac{\left\langle A_{0}^{\frac{1}{2}} \pi_{h} \psi, A_{0}^{\frac{1}{2}} \psi_{h}\right\rangle}{\left\|\psi_{h}\right\|} \\
& =\sup _{\psi_{h} \in \mathcal{V}_{h}} \frac{\left\langle A_{0}^{\frac{1}{2}}\left(\pi_{h} \psi-\psi\right), A_{0}^{\frac{1}{2}} \psi_{h}\right\rangle+\left\langle A_{0} \psi, \psi_{h}\right\rangle}{\left\|\psi_{h}\right\|} \\
& \leq\left\|\pi_{h} \psi-\psi\right\|_{\mathcal{D}\left(A_{0}^{\frac{1}{2}}\right)} \sup _{\psi_{h} \in \mathcal{V}_{h}} \frac{\left\|\psi_{h}\right\|_{\mathcal{D}\left(A_{0}^{\frac{1}{2}}\right)}}{\left\|\psi_{h}\right\|}+\|\psi\|_{\mathcal{D}\left(A_{0}\right)} \\
& \leq C h^{\theta-1}\|\psi\|_{\mathcal{D}\left(A_{0}\right)}+\|\psi\|_{\mathcal{D}\left(A_{0}\right)}
\end{aligned}
$$

using (2.9) and (2.6). Since $\theta \geq 1$ we infer the first bound $\left\|A_{0 h} \pi_{h} \psi\right\| \leq C\|\psi\|_{\mathcal{D}\left(A_{0}\right)}$, while the second bound $\left\|A_{0 h} \check{\pi}_{h} \varphi\right\|_{\mathcal{D}\left(A_{0}^{\frac{1}{2}}\right)} \leq C\|\varphi\|_{\mathcal{D}\left(A_{0}^{\frac{3}{2}}\right)}$ is obtained by similar albeit simpler - arguments. 
Therefore, the major difficulty in devising a discretization scheme in the framework of Th. 2.1 consists in devising an observation operator that satisfies the observability property (2.3), see Sec. 5 and Sec. 6 for examples and corresponding numerical assessments. This condition being ascertained, the "closed-loop" discretization (2.20) thus provides a much more effective strategy - for arbitrary simulation times - than the direct discretization approach. In order to prove our result we will use some uniform discrete observability bounds which - as we establish in the next section - are automatically satisfied under the assumptions of the theorem.

\section{Uniform observability of the spatial semi-discrete system}

We consider the following spatial semi-discretization of the system (2.1)

$$
\left\{\begin{array}{l}
\ddot{w}_{h}(t)+A_{0 h} w_{h}(t)=0, \quad t>0 \\
w_{h}(0)=w_{0 h}, \quad \dot{w}_{h}(0)=w_{1 h}
\end{array}\right.
$$

with $w_{0 h}, w_{1 h} \in \mathcal{V}_{h}$.

The aim of this section is to establish a discrete counterpart of the observability property (2.3), obtained by extending the result given in Th. 8.1 of Ref. 10 to the case of operators $H_{0} \in \mathcal{L}\left(\mathcal{D}\left(A_{0}^{\frac{1}{2}}\right), \mathcal{Z}\right)$ using the characterization of observability by resolvent inequalities, see also Refs. 23, 25, 32. We further point out that the case considered here is also addressed in the coincidental, independent, work of Ref. 26 . The following proposition states our discrete observability result.

Proposition 3.1. Let $A_{0}$ be a self-adjoint positive definite operator with compact resolvent and $H_{0} \in \mathcal{L}\left(\mathcal{D}\left(A_{0}^{\frac{1}{2}}\right), \mathcal{Z}\right)$. Assume that (2.6)-(2.11) are satisfied, and that System (2.1)-(2.2) is exactly observable. Then there exist $\eta>0, k^{*}>0, T^{*}>0$ such that any solution of (3.1) with

$$
\left(w_{0 h}, w_{1 h}\right) \in\left(\mathcal{C}_{h}\left(\eta / h^{\theta}\right)\right)^{2}
$$

satisfies

$$
\int_{0}^{T^{*}}\left\|H_{0 h} w_{h}(t)\right\|_{\mathcal{Z}}^{2} d t \geq k^{*}\left(\left\|A_{0 h}^{\frac{1}{2}} w_{0 h}\right\|^{2}+\left\|w_{1 h}\right\|^{2}\right) .
$$

We will need the following preliminary results, see also Ref. 10.

Lemma 3.1. For any $\phi_{h} \in \mathcal{V}_{h}$, define $\Phi_{h} \in \mathcal{D}\left(A_{0}\right)$ such that

$$
A_{0} \Phi_{h}=A_{0 h} \phi_{h} .
$$

Then,

$$
\begin{gathered}
\left\|\phi_{h}-\Phi_{h}\right\| \leq C h^{\theta}\left\|\phi_{h}\right\|_{\mathcal{D}\left(A_{0}^{\frac{1}{2}}\right)}, \\
\left\|\phi_{h}-\Phi_{h}\right\|_{\mathcal{D}\left(A_{0}^{\frac{1}{2}}\right)} \leq C h^{\theta}\left\|A_{0 h} \phi_{h}\right\|,
\end{gathered}
$$




$$
\left|\left\|A_{0 h}^{\frac{1}{2}} \phi_{h}\right\|^{2}-\left\|A_{0}^{\frac{1}{2}} \Phi_{h}\right\|^{2}\right| \leq\left\|A_{0 h} \phi_{h}\right\|\left\|\phi_{h}-\Phi_{h}\right\| .
$$

Proof. By the definition of $\Phi_{h}$ and $A_{0 h}$ we have

$$
\left\langle A_{0}^{\frac{1}{2}} \phi_{h}, A_{0}^{\frac{1}{2}} v\right\rangle=\left\langle A_{0}^{\frac{1}{2}} \Phi_{h}, A_{0}^{\frac{1}{2}} v\right\rangle, \quad \forall v \in \mathcal{V}_{h},
$$

namely, $\phi_{h}=\check{\pi}_{h} \Phi_{h}$. Therefore, by $(2.11)$

$$
\left\|\phi_{h}-\Phi_{h}\right\|_{\mathcal{D}\left(A_{0}^{\frac{1}{2}}\right)} \leq C h^{\theta}\left\|A_{0} \Phi_{h}\right\|=C h^{\theta}\left\|A_{0 h} \phi_{h}\right\|
$$

Moreover,

$$
\begin{aligned}
\left\|\Phi_{h}\right\|_{\mathcal{D}\left(A_{0}^{\frac{1}{2}}\right)} & =\sup _{v \in \mathcal{D}\left(A_{0}^{\frac{1}{2}}\right)} \frac{\left\langle A_{0} \Phi_{h}, v\right\rangle}{\|v\|_{\mathcal{D}\left(A_{0}^{\frac{1}{2}}\right)}}=\sup _{v \in \mathcal{D}\left(A_{0}^{\frac{1}{2}}\right)} \frac{\left\langle A_{0 h}^{\frac{1}{2}} \phi_{h}, A_{0 h}^{\frac{1}{2}} \pi_{h} v\right\rangle}{\|v\|_{\mathcal{D}\left(A_{0}^{\frac{1}{2}}\right)}} \\
& \leq\left\|\phi_{h}\right\|_{\mathcal{D}\left(A_{0}^{\frac{1}{2}}\right)} \sup _{v \in \mathcal{D}\left(A_{0}^{\frac{1}{2}}\right)} \frac{\left\|\pi_{h} v\right\|_{\mathcal{D}\left(A_{0}^{\frac{1}{2}}\right)}}{\|v\|_{\mathcal{D}\left(A_{0}^{\frac{1}{2}}\right)}} \\
& \leq C\left\|\phi_{h}\right\|_{\mathcal{D}\left(A_{0}^{\frac{1}{2}}\right)}
\end{aligned}
$$

due to (2.7), and then (3.4) immediately follows by applying (2.10) with $\Phi_{h}$. Next,

$$
\left\|A_{0 h}^{\frac{1}{2}} \phi_{h}\right\|^{2}-\left\|A_{0}^{\frac{1}{2}} \Phi_{h}\right\|^{2}=\left\langle A_{0 h} \phi_{h}, \phi_{h}\right\rangle-\left\langle A_{0} \Phi_{h}, \Phi_{h}\right\rangle=\left\langle A_{0 h} \phi_{h}, \phi_{h}-\Phi_{h}\right\rangle,
$$

and we conclude.

We are now in the position to prove the result of Prop. 3.1.

Proof. [Prop. 3.1] The exact observability of System (2.1)-(2.2) is equivalent to the existence of two positive constants $m$ and $M$ such that the following resolvent estimate is satisfied

$$
\left\|A_{0}^{\frac{1}{2}} \phi\right\|^{2} \leq M^{2}\left\|\left(A_{0}-\lambda I\right) \phi\right\|^{2}+m^{2}\left\|H_{0} \phi\right\|^{2}, \quad \forall \phi \in \mathcal{D}\left(A_{0}\right), \quad \forall \lambda>0,
$$

see Th. 8.2 of Ref. 10. The idea of the proof is then to show that there exist $\eta>0$, $m_{*}>0$, and $M_{*}>0$ such that

$$
\begin{aligned}
\left\|A_{0 h}^{\frac{1}{2}} \phi_{h}\right\|^{2} \leq M_{*}^{2}\left\|\left(A_{0 h}-\lambda I\right) \phi_{h}\right\|^{2}+ & m_{*}^{2}\left\|H_{0 h} \phi_{h}\right\|_{\mathcal{Z}}^{2} \\
& \forall \phi_{h} \in \mathcal{C}_{h}\left(\eta / h^{\theta}\right), \quad \forall \lambda \in\left(0, \eta / h^{\theta}\right],
\end{aligned}
$$

where $\eta>0$ is a parameter independent of $h$ and to be specified later. As explained in Ref. 10 the observability estimate (3.3) will then follow.

In order to obtain (3.8) we adapt the proof of Th. 1.1 in Ref. 10. For an arbitrary $\phi_{h} \in \mathcal{V}_{h}$, we introduce $\Phi_{h}$ as in the proof of Lem. 3.1 above, and applying (3.7) yields

$$
\left\|A_{0}^{\frac{1}{2}} \Phi_{h}\right\|^{2} \leq M^{2}\left\|\left(A_{0}-\lambda I\right) \Phi_{h}\right\|^{2}+m^{2}\left\|H_{0} \Phi_{h}\right\|^{2}, \quad \forall \lambda>0 .
$$


We have

$$
\left(A_{0}-\lambda I\right) \Phi_{h}=\left(A_{0 h}-\lambda I\right) \phi_{h}+\lambda\left(\phi_{h}-\Phi_{h}\right),
$$

hence,

$$
\left\|\left(A_{0}-\lambda I\right) \Phi_{h}\right\|^{2} \leq 2\left\|\left(A_{0 h}-\lambda I\right) \phi_{h}\right\|^{2}+2 \lambda^{2}\left\|\phi_{h}-\Phi_{h}\right\|^{2} .
$$

Also,

$$
H_{0} \Phi_{h}=H_{0 h} \phi_{h}+H_{0}\left(\phi_{h}-\Phi_{h}\right),
$$

with

$$
\left\|H_{0}\left(\phi_{h}-\Phi_{h}\right)\right\|_{\mathcal{Z}} \leq\left\|H_{0}\right\|_{\mathcal{L}\left(\mathcal{D}\left(A_{0}^{\frac{1}{2}}\right), \mathcal{Z}\right)}\left\|\phi_{h}-\Phi_{h}\right\|_{\mathcal{D}\left(A_{0}^{\frac{1}{2}}\right)}
$$

which implies

$$
\left\|H_{0} \Phi_{h}\right\|_{\mathcal{Z}}^{2} \leq 2\left\|H_{0 h} \phi_{h}\right\|_{\mathcal{Z}}^{2}+2\left\|H_{0}\right\|_{\mathcal{L}\left(\mathcal{D}\left(A_{0}^{\frac{1}{2}}\right), \mathcal{Z}\right)}^{2}\left\|\phi_{h}-\Phi_{h}\right\|_{\mathcal{D}\left(A_{0}^{\frac{1}{2}}\right)}^{2} .
$$

Note that, if $\phi_{h} \in \mathcal{C}_{h}\left(\eta / h^{\theta}\right)$, we have $\left\|A_{0 h} \phi_{h}\right\| \leq \eta^{\frac{1}{2}} h^{-\frac{\theta}{2}}\left\|A_{0 h}^{\frac{1}{2}} \phi_{h}\right\|$. With this, gathering now (3.6), (3.10) and (3.11), and recalling (3.4)-(3.5), we get for any $\phi_{h} \in \mathcal{C}_{h}\left(\eta / h^{\theta}\right)$ and any $\lambda \in\left(0, \eta / h^{\theta}\right]$

$$
\left\{\begin{array}{l}
\left\|A_{0 h}^{\frac{1}{2}} \phi_{h}\right\|^{2} \leq\left\|A_{0}^{\frac{1}{2}} \Phi_{h}\right\|^{2}+C \eta^{\frac{1}{2}} h^{\frac{\theta}{2}}\left\|A_{0 h}^{\frac{1}{2}} \phi_{h}\right\|^{2} \\
\left\|\left(A_{0}-\lambda I\right) \Phi_{h}\right\|^{2} \leq 2\left\|\left(A_{0 h}-\lambda I\right) \phi_{h}\right\|^{2}+C \eta^{2}\left\|A_{0 h}^{\frac{1}{2}} \phi_{h}\right\|^{2} \\
\left\|H_{0} \Phi_{h}\right\|_{\mathcal{Z}}^{2} \leq 2\left\|H_{0 h} \phi_{h}\right\|_{\mathcal{Z}}^{2}+C \eta h^{\theta}\left\|A_{0 h}^{\frac{1}{2}} \phi_{h}\right\|^{2}
\end{array}\right.
$$

We can substitute these inequalities in (3.9), which gives with $h \leq 1$

$$
\left(1-C\left(\eta^{\frac{1}{2}}+\eta+\eta^{2}\right)\right)\left\|A_{0 h}^{\frac{1}{2}} \phi_{h}\right\|^{2} \leq 2 M^{2}\left\|\left(A_{0 h}-\lambda I\right) \phi_{h}\right\|^{2}+2 m^{2}\left\|H_{0 h} \phi_{h}\right\|_{\mathcal{Z}}^{2} .
$$

Finally, there is no difficulty in setting $\eta$ - independently of $h$ - so that (3.8) holds.

Remark 3.1 (Resolvent conditions). In fact, in Th. 8.2 of Ref. 10 the resolvent inequalities (3.7) and (3.8) are stated for $\lambda$ in the convex hull of the spectrum, but they are also shown to be equivalent to the corresponding inequalities taken for $\lambda>0$.

\section{Numerical analysis of the "closed-loop" discretization scheme}

The objective of this section is to establish the error estimate given in Th. 2.1. To that end, we will use a stability-consistency approach in which the stability argument is essentially based on the discrete observability property proven in the previous section. Denoting

$$
E_{h}^{k}=\bar{x}_{h}^{k}-\Pi_{h} x(k \Delta t)
$$


the idea of the proof is - indeed - to show that $E_{h}^{k}$ satisfies a discrete system with exponentially stable dynamics fed by well-controlled consistency error terms. Introducing also

$$
\widetilde{E}_{h}^{k}=E_{h}^{k}-\varepsilon \Delta t V_{\varepsilon} E_{h}^{k}
$$

we have

$$
\begin{aligned}
& \frac{\widetilde{E}_{h}^{k+1}-E_{h}^{k}}{\Delta t}=\frac{E_{h}^{k+1}-\varepsilon \Delta t V_{\varepsilon} E_{h}^{k+1}-E_{h}^{k}}{\Delta t} \\
& =\frac{\bar{x}_{h}^{k+1}-\Pi_{h} x((k+1) \Delta t)-\varepsilon \Delta t V_{\varepsilon}\left(\bar{x}_{h}^{k+1}-\Pi_{h} x((k+1) \Delta t)\right)-\bar{x}_{h}^{k}+\Pi_{h} x(k \Delta t)}{\Delta t} \\
& =\frac{\bar{x}_{h}^{k+1}-\varepsilon \Delta t V_{\varepsilon} \bar{x}_{h}^{k+1}-\bar{x}_{h}^{k}}{\Delta t}-\Pi_{h} \frac{x((k+1) \Delta t)-x(k \Delta t)}{\Delta t}-\varepsilon V_{\varepsilon} \Pi_{h} x((k+1) \Delta t) \\
& =\frac{\widetilde{x}_{h}^{k+1}-\bar{x}_{h}^{k}}{\Delta t}-\Pi_{h} \frac{\dot{x}(k \Delta t)+\dot{x}((k+1) \Delta t)}{2}+\Delta t O_{h}^{k}-\varepsilon V_{\varepsilon} \Pi_{h} x((k+1) \Delta t),
\end{aligned}
$$

where $O_{h}^{k}$ is a term coming from the first-order finite difference approximation of $\dot{x}(k \Delta t)$ and remains bounded in $\mathcal{X}$ with respect to $h$ and $k$. Indeed, from classical semigroup theory results applied to $A: \mathcal{D}\left(A^{2}\right) \rightarrow \mathcal{D}(A)$, we have that $x_{0} \in \mathcal{D}\left(A^{2}\right)=$ $\mathcal{D}\left(A_{0}^{\frac{3}{2}}\right) \times \mathcal{D}\left(A_{0}\right)$ implies $^{31,38}$

$$
x \in C\left([0, \infty), \mathcal{D}\left(A^{2}\right)\right) \cap C^{1}([0, \infty), \mathcal{D}(A)),
$$

and moreover the conservative nature of the system can be invoked with

$$
A^{2} x=-\left(\begin{array}{cc}
A_{0} & 0 \\
0 & A_{0}
\end{array}\right) x=\ddot{x},
$$

to infer that we also have $x \in C^{2}([0, \infty), \mathcal{X})$, and

$$
\|\ddot{x}\|_{\mathcal{X}}=\left\|A^{2} x_{0}\right\|_{\mathcal{X}}=\left\|x_{0}\right\|_{\mathcal{D}\left(A^{2}\right)}
$$

shows that $\ddot{x}$ is uniformly bounded in $\mathcal{X}=\mathcal{D}\left(A_{0}^{\frac{1}{2}}\right) \times \mathcal{H}$.

Next, from (2.20) and (2.4) we obtain

$$
\begin{aligned}
& \frac{\widetilde{E}_{h}^{k+1}-E_{h}^{k}}{\Delta t}=A_{h} \frac{\bar{x}_{h}^{k}+\widetilde{\bar{x}}_{h}^{k+1}}{2}+\gamma H_{h}^{*}\left(\frac{z^{k}+z^{k+1}}{2}-H_{h} \frac{\bar{x}_{h}^{k}+\widetilde{\bar{x}}_{h}^{k+1}}{2}\right) \\
&- \Pi_{h} A \frac{x(k \Delta t)+x((k+1) \Delta t)}{2}+\Delta t O_{h}^{k}-\varepsilon V_{\varepsilon} \Pi_{h} x((k+1) \Delta t) \\
&=A_{h} \frac{E_{h}^{k}+\widetilde{E}_{h}^{k+1}}{2}+A_{h} \frac{\Pi_{h} x(k \Delta t)+\Pi_{h} x((k+1) \Delta t)-\varepsilon \Delta t V_{\varepsilon} \Pi_{h} x((k+1) \Delta t)}{2} \\
&-\gamma H_{h}^{*} H_{h} \frac{\bar{x}_{h}^{k}+\widetilde{\bar{x}}_{h}^{k+1}-\Pi_{h} x(k \Delta t)-\Pi_{h} x((k+1) \Delta t)}{2} \\
&+\gamma H_{h}^{*} H\left(I-\Pi_{h}\right) \frac{x(k \Delta t)+x((k+1) \Delta t)}{2} \\
&-\Pi_{h} A \frac{x(k \Delta t)+x((k+1) \Delta t)}{2}+\Delta t O_{h}^{k}-\varepsilon V_{\varepsilon} \Pi_{h} x((k+1) \Delta t)
\end{aligned}
$$


March 1, 2012 13:17 WSPC/INSTRUCTION FILE waveSDFNumAnalm3as

Hence,

$$
\begin{aligned}
& \frac{\widetilde{E}_{h}^{k+1}-E_{h}^{k}}{\Delta t}=A_{h} \frac{E_{h}^{k}+\widetilde{E}_{h}^{k+1}}{2}-\gamma H_{h}^{*} H_{h} \frac{E_{h}^{k}+\widetilde{E}_{h}^{k+1}}{2} \\
& +A_{h} \frac{\Pi_{h} x(k \Delta t)+\Pi_{h} x((k+1) \Delta t)-\varepsilon \Delta t V_{\varepsilon} \Pi_{h} x((k+1) \Delta t)}{2} \\
& +\varepsilon \gamma \Delta t / 2 H_{h}^{*} H_{h} V_{\varepsilon} \Pi_{h} x((k+1) \Delta t) \\
& +\gamma H_{h}^{*} H\left(I-\Pi_{h}\right) \frac{x(k \Delta t)+x((k+1) \Delta t)}{2} \\
& -\Pi_{h} A \frac{x(k \Delta t)+x((k+1) \Delta t)}{2}+\Delta t O_{h}^{k}-\varepsilon V_{\varepsilon} \Pi_{h} x((k+1) \Delta t) \\
& =A_{h} \frac{E_{h}^{k}+\widetilde{E}_{h}^{k+1}}{2}-\gamma H_{h}^{*} H_{h} \frac{E_{h}^{k}+\widetilde{E}_{h}^{k+1}}{2} \\
& +\left(A_{h} \Pi_{h}-\Pi_{h} A\right) \frac{x(k \Delta t)+x((k+1) \Delta t)}{2} \\
& +\gamma H_{h}^{*} H\left(I-\Pi_{h}\right) \frac{x(k \Delta t)+x((k+1) \Delta t)}{2} \\
& +\left(-\frac{\Delta t}{2} A_{h}+\frac{\Delta t \gamma}{2} H_{h}^{*} H_{h}-1\right) \varepsilon V_{\varepsilon} \Pi_{h} x((k+1) \Delta t)+\Delta t O_{h}^{k} .
\end{aligned}
$$

Then, denoting

$$
\begin{aligned}
G_{h}^{k}= & \left(A_{h} \Pi_{h}-\Pi_{h} A\right) \frac{x(k \Delta t)+x((k+1) \Delta t)}{2} \\
& +\gamma H_{h}^{*} H\left(I-\Pi_{h}\right) \frac{x(k \Delta t)+x((k+1) \Delta t)}{2} \\
& +\left(-\frac{\Delta t}{2} A_{h}+\frac{\Delta t \gamma}{2} H_{h}^{*} H_{h}-1\right) \varepsilon V_{\varepsilon} \Pi_{h} x((k+1) \Delta t)+\Delta t O_{h}^{k},
\end{aligned}
$$

$E_{h}^{k}$ satisfies

$$
\left\{\begin{array}{l}
\frac{\widetilde{E}_{h}^{k+1}-E_{h}^{k}}{\Delta t}=A_{h} \frac{E_{h}^{k}+\widetilde{E}_{h}^{k+1}}{2}-\gamma H_{h}^{*} H_{h} \frac{E_{h}^{k}+\widetilde{E}_{h}^{k+1}}{2}+G_{h}^{k} \\
\frac{E_{h}^{k+1}-\widetilde{E}_{h}^{k+1}}{\Delta t}=\varepsilon V_{\varepsilon} E_{h}^{k+1} \\
E_{h}^{0}=0
\end{array}\right.
$$

Lemma 4.1. Under the assumptions of Th. 2.1, there exists a constant $C>0$, independent of $h$ and $\Delta t$, such that the solution of (4.1) satisfies

$$
\left\|E_{h}^{n}\right\|_{\mathcal{X}} \leq C \max _{0 \leq k<n}\left\|G_{h}^{k}\right\|_{\mathcal{X}}, \quad \forall n \in \mathbb{N}^{*}
$$

Proof. Let $\left(F_{h}^{k}, \widetilde{F}_{h}^{k}\right)$ be the solution of the following discrete homogeneous system

$$
\left\{\begin{array}{l}
\frac{\widetilde{F}_{h}^{k+1}-F_{h}^{k}}{\Delta t}=A_{h} \frac{F_{h}^{k}+\widetilde{F}_{h}^{k+1}}{2}-\gamma H_{h}^{*} H_{h} \frac{F_{h}^{k}+\widetilde{F}_{h}^{k+1}}{2} \\
\frac{F_{h}^{k+1}-\widetilde{F}_{h}^{k+1}}{\Delta t}=\varepsilon V_{\varepsilon} F_{h}^{k+1}
\end{array}\right.
$$


Combining the assumptions of Th. 2.1 with the uniform observability result for truncated initial data given by Prop. 3.1, all the assumptions of Th. 3.7 in Ref. 13 are fulfilled. Therefore, the latter provides two positive constants $M_{0}$ and $\mu_{0}$ such that

$$
\left\|F_{h}^{k}\right\|_{\mathcal{X}} \leq M_{0} e^{-\mu_{0} k \Delta t}\left\|F_{h}^{0}\right\|_{\mathcal{X}}
$$

We can easily see that $F_{h}^{k}$ can be explicitly computed as

$$
F_{h}^{k}=\mathcal{Q}_{1} \mathcal{Q}_{2} F_{h}^{k-1}=\left(\mathcal{Q}_{1} \mathcal{Q}_{2}\right)^{k} F_{h}^{0},
$$

where we denote

$$
\mathcal{Q}_{1}=\left(I-\varepsilon \Delta t V_{\varepsilon}\right)^{-1}\left[I-\frac{\Delta t}{2}\left(A_{h}-\gamma H_{h}^{*} H_{h}\right)\right]^{-1}, \quad \mathcal{Q}_{2}=I+\frac{\Delta t}{2}\left(A_{h}-\gamma H_{h}^{*} H_{h}\right) .
$$

Substituting (4.5) in (4.4), we obtain that

$$
\left\|\left(\mathcal{Q}_{1} \mathcal{Q}_{2}\right)^{k} F_{h}^{0}\right\|_{\mathcal{X}} \leq M_{0} e^{-\mu_{0} k \Delta t}\left\|F_{h}^{0}\right\|_{\mathcal{X}}, \quad \forall k \in \mathbb{N}^{*}, \quad F_{h}^{0} \in \mathcal{V}_{h}
$$

With this notation, the solution of (4.1) is given by

$$
E_{h}^{n}=\left(\mathcal{Q}_{1} \mathcal{Q}_{2}\right)^{n} E_{h}^{0}+\Delta t \sum_{i=0}^{n-1}\left(\mathcal{Q}_{1} \mathcal{Q}_{2}\right)^{i} \mathcal{Q}_{1} G_{h}^{n-1-i} .
$$

Since both semigroups generated by $\left(A_{h}-\gamma H_{h}^{*} H_{h}\right)$ and by $V_{\varepsilon}$ are contraction semigroups, using e.g. Corollary 2.3 .3 in Ref. 38 we easily have that $\left\|Q_{1}\right\|_{\mathcal{L}\left(\mathcal{X}_{h}\right)} \leq 1$. Therefore, combining $(4.1)_{3},(4.7)$ and (4.8), we infer

$$
\begin{aligned}
\left\|E_{h}^{n}\right\|_{\mathcal{X}} & \leq \Delta t \sum_{i=0}^{n-1} M_{0} e^{-\mu_{0} i \Delta t}\left\|G_{h}^{n-1-i}\right\|_{\mathcal{X}} \leq M_{0} \max _{0 \leq i<n}\left\{\left\|G_{h}^{i}\right\|_{\mathcal{X}}\right\} \Delta t \sum_{i=0}^{n-1}\left(e^{-\mu_{0} \Delta t}\right)^{i} \\
& \leq M_{0} \max _{0 \leq i<n}\left\{\left\|G_{h}^{i}\right\|_{\mathcal{X}}\right\} \frac{\Delta t}{1-e^{-\mu_{0} \Delta t}} .
\end{aligned}
$$

Since $0<\Delta t \leq \Delta t^{*}$, it is elementary to deduce that

$$
\left\|E_{h}^{n}\right\|_{\mathcal{X}} \leq \frac{M_{0} \Delta t^{*}}{1-e^{-\mu_{0} \Delta t^{*}}} \max _{0 \leq i<n}\left\{\left\|G_{h}^{i}\right\|_{\mathcal{X}}\right\}
$$

which completes the proof of the lemma.

We are now in a position to prove Th. 2.1. Considering the term $A_{h} \Pi_{h}-\Pi_{h} A$ contained in $G_{h}^{k}$, for every $\left(\begin{array}{l}\varphi \\ \psi\end{array}\right) \in \mathcal{D}\left(A_{0}^{\frac{3}{2}}\right) \times \mathcal{D}\left(A_{0}\right)$ and $\left(\begin{array}{l}\varphi_{h} \\ \psi_{h}\end{array}\right) \in \mathcal{X}_{h}$, we have, on the one hand,

$$
\begin{aligned}
\left\langle A_{h} \Pi_{h}\left(\begin{array}{c}
\varphi \\
\psi
\end{array}\right),\left(\begin{array}{c}
\varphi_{h} \\
\psi_{h}
\end{array}\right)\right\rangle_{\mathcal{X}} & =\left\langle\left(\begin{array}{c}
\pi_{h} \psi \\
-A_{0 h} \check{\pi}_{h} \varphi
\end{array}\right),\left(\begin{array}{c}
\varphi_{h} \\
\psi_{h}
\end{array}\right)\right\rangle_{\mathcal{X}} \\
& =\left\langle\pi_{h} \psi, \varphi_{h}\right\rangle_{\mathcal{D}\left(A_{0}^{\frac{1}{2}}\right)}-\left\langle A_{0 h} \check{\pi}_{h} \varphi, \psi_{h}\right\rangle \\
& =\left\langle\pi_{h} \psi, \varphi_{h}\right\rangle_{\mathcal{D}\left(A_{0}^{\frac{1}{2}}\right)}-\left\langle A_{0}^{\frac{1}{2}} \check{\pi}_{h} \varphi, A_{0}^{\frac{1}{2}} \psi_{h}\right\rangle \\
& =\left\langle\pi_{h} \psi, \varphi_{h}\right\rangle_{\mathcal{D}\left(A_{0}^{\frac{1}{2}}\right)}-\left\langle A_{0}^{\frac{1}{2}} \varphi, A_{0}^{\frac{1}{2}} \psi_{h}\right\rangle
\end{aligned}
$$


On the other hand,

$$
\begin{aligned}
\left\langle\Pi_{h} A\left(\begin{array}{c}
\varphi \\
\psi
\end{array}\right),\left(\begin{array}{c}
\varphi_{h} \\
\psi_{h}
\end{array}\right)\right\rangle_{\mathcal{X}} & =\left\langle\left(\begin{array}{c}
\check{\pi}_{h} \psi \\
-\pi_{h} A_{0} \varphi
\end{array}\right),\left(\begin{array}{c}
\varphi_{h} \\
\psi_{h}
\end{array}\right)\right\rangle_{\mathcal{X}}=\left\langle\check{\pi}_{h} \psi, \varphi_{h}\right\rangle_{\mathcal{D}\left(A_{0}^{\frac{1}{2}}\right)}-\left\langle\pi_{h} A_{0} \varphi, \psi_{h}\right\rangle \\
& =\left\langle\psi, \varphi_{h}\right\rangle_{\mathcal{D}\left(A_{0}^{\frac{1}{2}}\right)}-\left\langle A_{0}^{\frac{1}{2}} \varphi, A_{0}^{\frac{1}{2}} \psi_{h}\right\rangle .
\end{aligned}
$$

Therefore,

$$
\begin{aligned}
\left\|\left(A_{h} \Pi_{h}-\Pi_{h} A\right)\left(\begin{array}{c}
\varphi \\
\psi
\end{array}\right)\right\|_{\mathcal{X}} & =\sup _{\left(\begin{array}{c}
\varphi_{h} \\
\psi_{h}
\end{array}\right) \in \mathcal{X}_{h},\left\|\left(\begin{array}{c}
\varphi_{h} \\
\psi_{h}
\end{array}\right)\right\|_{\mathcal{X}} \leq 1}\left|\left\langle A_{h} \Pi_{h}-\Pi_{h} A\right)\left(\begin{array}{c}
\varphi \\
\psi
\end{array}\right),\left(\begin{array}{c}
\varphi_{h} \\
\psi_{h}
\end{array}\right)\right\rangle_{\mathcal{X}} \mid \\
= & \sup _{\left(\begin{array}{c}
\varphi_{h} \\
\psi_{h}
\end{array}\right) \in \mathcal{X}_{h},\left\|\left(\begin{array}{c}
\varphi_{h} \\
\psi_{h}
\end{array}\right)\right\|_{\mathcal{X}} \leq 1}\left|\left\langle\pi_{h} \psi-\psi, \varphi_{h}\right\rangle_{\mathcal{D}\left(A_{0}^{\frac{1}{2}}\right)}\right| \\
\leq & \left\|A_{0}^{\frac{1}{2}}\left(\pi_{h} \psi-\psi\right)\right\| \\
\leq & C_{0} h^{\theta}\|\psi\|_{\mathcal{D}\left(A_{0}\right),}
\end{aligned}
$$

using (2.9). Hence, from the definition of $G_{h}^{k}$ and the bounds (2.19) and (4.9), there exists a constant $C_{0}\left(x_{0}\right)$ such that

$$
\left\|G_{h}^{k}\right\|_{\mathcal{X}} \leq C_{0}\left(x_{0}\right) \max \left(\varepsilon, \varepsilon^{2} h^{-1} \Delta t\right), \quad \forall k \in \mathbb{N} .
$$

Note in particular that the last term in the right-hand side comes from combining (2.19) with

$$
\left\|A_{h}\left(\begin{array}{c}
\varphi_{h} \\
\psi_{h}
\end{array}\right)\right\|_{\mathcal{X}}=\left\|\left(\begin{array}{c}
\psi_{h} \\
A_{0 h} \varphi_{h}
\end{array}\right)\right\|_{\mathcal{X}} \leq C h^{-1}\left\|\left(\begin{array}{c}
\varphi_{h} \\
\psi_{h}
\end{array}\right)\right\|_{\mathcal{X}}
$$

where we used the inverse inequality (2.6). Finally, combining (4.10) and the result of the Lem. 4.1, we obtain the conclusion of Th. 2.1.

Remark 4.1 (Time discretization sharpness). The error estimate appears to be sub-optimal with respect to time discretization since the mid-point time scheme in itself could be expected to be of second-order. This may be improved by revisiting the work of Ref. 13 with a different weight on the viscosity operator. Note that Newmark type discretizations are also considered with observers in Ref. 11, albeit in the context of frequency-truncated initial conditions.

Remark 4.2 (Case of available velocity measurements). We emphasize that the case of a measurement operator $H$ applying on the second part of the state variable - namely, on $\dot{w}$ - would be much more straightforward to analyze using Ref. 13 and more standard observability inequalities, and would lead to similar error estimates for the discretization. Here, we focused on measurements of $w$ itself primarily because they are often more readily available in practice, and of course time-differentiating such data is frequently very detrimental due to measurement errors. Nevertheless, in some instances measurements on the time derivatives e.g. velocities - are directly available, in which case the observer approach can be 
effectively used, see also Refs. 28, 15. Moreover, this clearly extends to separate simultaneous measurements of $w$ and $\dot{w}$, namely, with observation operators of the type

$$
H=\left(\begin{array}{cc}
H_{0} & 0 \\
0 & H_{1}
\end{array}\right)
$$

since the observability contributions provided by both sub-parts $H_{0}$ and $H_{1}$ then add up in the required global observability inequality.

Remark 4.3 (Measurement noise). Measurement errors would typically directly enter the error system (4.1) as additional terms in $G_{h}^{k}$, hence could be easily included in the above numerical analysis, see Ref. 29 for detailed related numerical assessments. This holds in particular for errors arising from the measurement time sampling, which in practice is likely to be coarser than the computational time step, and which can be handled by interpolating the measurements at all (computational) time steps, leading to straightforward interpolation errors. This also further illustrates how time-differentiating measurements on $w$ as a substitute for measurements on $\dot{w}$ would enter the error system in the form of amplified measurement errors.

\section{Application to the wave equation}

Let $\Omega \subset \mathbb{R}^{n}$ be a bounded open connected set with a Lipschitz boundary $\partial \Omega$ and let $\omega \subset \Omega$ be an open and non-empty subset of $\Omega$. We consider the following wave equation

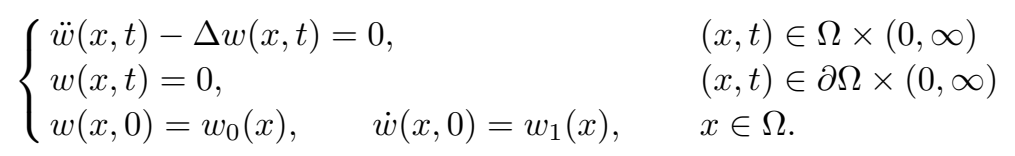

The aim of this section is to apply the discretization method proposed in (2.20) to the wave equation, considering as measured data

$$
z(t)=\left.w(\cdot, t)\right|_{\omega}, \quad t \geq 0 .
$$

To that purpose, the careful definition of an observation operator and the choice of the associated $\mathcal{Z}$-norm are crucial, ${ }^{29}$ and we follow the original construction of Ref. 5 to introduce the operator

$$
\mathcal{L}_{\omega}: H^{1}(\omega) \rightarrow H_{0}^{1}(\Omega), \quad \mathcal{L}_{\omega} \phi=\psi,
$$

such that $\psi$ is the solution of the following elliptic equation

$$
\begin{cases}\Delta \psi=0, & \text { in } \Omega \backslash \omega \\ \psi=0, & \text { on } \partial \Omega \\ \psi=\phi, & \text { in } \bar{\omega}\end{cases}
$$

namely, a harmonic lifting operator. In what follows, we recast the wave equation (5.1) in the abstract formalism of Section 2. 
Denote $\mathcal{H}=L^{2}(\Omega)$ and let $A_{0}: \mathcal{D}\left(A_{0}\right) \rightarrow \mathcal{H}$ be the Dirichlet Laplacian, i.e.,

$$
A_{0} \varphi=-\Delta \varphi, \quad \mathcal{D}\left(A_{0}\right)=\left\{\varphi \in H_{0}^{1}(\Omega) \mid \Delta \varphi \in L^{2}(\Omega)\right\},
$$

and $\mathcal{D}\left(A_{0}\right)=H^{2}(\Omega) \cap H_{0}^{1}(\Omega)$ under some regularity assumptions on the domain, e.g. $\partial \Omega$ of class $C^{2}$, see Ref. 14 . Moreover, it is easy to see that $\mathcal{D}\left(A_{0}^{\frac{1}{2}}\right)=H_{0}^{1}(\Omega)$. We now define the measurement space $\mathcal{Z}=H^{1}(\omega)$, equipped with the norm $\left\|\mathcal{L}_{\omega}(\cdot)\right\|_{H_{0}^{1}(\Omega)}$, which is equivalent to the usual norm of $H^{1}(\omega)$, see Ref. 5 . Therefore, we define the observation operator $H_{0} \in \mathcal{L}\left(\mathcal{D}\left(A_{0}^{\frac{1}{2}}\right), \mathcal{Z}\right)$ by

$$
H_{0} \varphi=\left.\varphi\right|_{\omega}, \quad \forall \varphi \in \mathcal{D}\left(A_{0}^{\frac{1}{2}}\right),
$$

which implies $H_{0}^{*}=\mathcal{L}_{\omega}$, since

$$
\begin{aligned}
\left\langle H_{0}^{*} \varphi, \zeta\right\rangle_{H_{0}^{1}(\Omega)}=\left\langle\varphi, H_{0} \zeta\right\rangle_{H^{1}(\omega)} & =\left\langle\varphi,\left.\zeta\right|_{\omega}\right\rangle_{H^{1}(\omega)} \\
& =\left\langle\mathcal{L}_{\omega} \varphi,\left.\mathcal{L}_{\omega} \zeta\right|_{\omega}\right\rangle_{H_{0}^{1}(\Omega)}=\left\langle\mathcal{L}_{\omega} \varphi, \zeta\right\rangle_{H_{0}^{1}(\Omega)}
\end{aligned}
$$

Clearly, with this notation, the equations (5.1)-(5.2) fit in the abstract form (2.1)(2.3). Moreover, Prop. 2.2 in Ref. 5 gives the exact observability of (5.1)-(5.2) - in the sense of Definition 2.1 - under a quite natural condition on the measurement domain, namely, the existence of a strict subset $\check{\omega} \subsetneq \omega$ and with $\operatorname{dist}(\Omega \backslash \omega, \check{\omega})>0$ such that the geometric control condition of Bardos, Lebeau and Rauch ${ }^{2}$ holds for $\check{\omega}$.

Remark 5.1 (Boundary measurements). In principle, the above construction of an observation operator by a harmonic lifting can be directly extended to boundary measurements of the main variable ${ }^{29}$ and the resulting operator $H^{*}$ is still bounded by similar arguments, in this case from the space of traces on the measured boundary to $H_{0}^{1}(\Omega)$. In such a case, however, counterexamples to the required observability condition are easily found - e.g. with analytical eigenmodes in domains of simple geometries, due to the fact that the boundary-normal derivative is not observed - which entails that the stabilized system is not asymptotically stable. Nevertheless, weaker stability may hold, and moreover this stabilization strategy can be effective with systems incorporating natural damping, see Ref. 29. Concerning other types of boundary measurements such as pertaining to velocity or boundary-normal derivatives, as discussed in Ref. 19 they generally correspond to cases for which proving stabilization by the observability condition is no longer the adequate strategy - although uniform stabilization can frequently be obtained by simple dissipative feedbacks - hence this lies beyond the scope of the present paper.

Assume now that $\left(\mathcal{V}_{h}\right)$ is a family of finite-dimensional spaces approximating $\mathcal{D}\left(A_{0}^{\frac{1}{2}}\right)$ and such that $(2.6)-(2.11)$ are satisfied for a given value of $\theta$, and let $A_{0 h}$ and $H_{0 h}$ be the corresponding discrete operators. As in Section 2 we also define the operators $A$ and $H$, with their discrete counterparts $A_{h}$ and $H_{h}$, and we choose as a viscosity operator $V_{\varepsilon}=\varepsilon A_{h}^{2}$ which satisfies the required conditions (2.17)(2.19) as already discussed. Therefore, all the assumptions of Th. 2.1 are fulfilled 
and, considering the numerical scheme given by (2.20), we have the uniform-in-time error estimate (2.21).

Of course, we can use spectral decomposition to write closed-form expressions of the solutions of the reference system. Since $A_{0}$ is a strictly positive, self-adjoint operator with compact resolvent, it has strictly positive eigenvalues $\lambda_{k}$ with $\lambda_{k} \rightarrow$ $\infty$, and there exists an orthonormal basis formed by the corresponding eigenvectors denoted by $\phi_{k}$. Decomposing the initial data in this basis

$$
w_{0}=\sum_{m=1}^{\infty} a_{m} \phi_{m}, \quad w_{1}=\sum_{m=1}^{\infty} b_{m} \phi_{m}, \quad \text { with } a_{m}, b_{m} \in \mathbb{R},
$$

the solution of $(5.1)$ is

$$
w(x, t)=\sum_{m=1}^{\infty}\left(a_{m} \cos \left(\sqrt{\lambda_{m}} t\right)+\frac{b_{m}}{\sqrt{\lambda_{m}}} \sin \left(\sqrt{\lambda_{m}} t\right)\right) \phi_{m}(x) .
$$

In the sequel we consider various examples of one- and two-dimensional wave equations, discretized with usual $\mathbb{P}_{1}$-Lagrange finite elements, which satisfy the conditions (2.6)-(2.11) with $\theta=1$ under standard assumptions for the meshes considered, see e.g. Ref. 3 (p.106). For each initial condition we first compute a reference solution given by:

- either the approximation of the exact solution obtained by truncating the spectral decomposition (5.6) at a controlled level for which the truncation error is negligible compared to the discretization error (when the eigenvalues and the eigenvectors of $A_{0}$ can be explicitly computed);

- or the solution of (2.20) with $\varepsilon=\gamma=0$ numerically computed for values of the discretization parameters $h$ and $\Delta t$ much smaller than for the assessed numerical solutions (when the eigenvalues and the eigenvectors of $A_{0}$ cannot be explicitly computed, as is the case for an arbitrary two-dimensional domain).

We then compare the interpolation of this reference solution in $\mathcal{V}_{h} \times \mathcal{V}_{h}$ - namely, obtained by computing the nodal values of the reference solution - with the solutions of (2.20) associated with the following choices of $\varepsilon$ and $\gamma$ :

- $\gamma=0, \varepsilon=0$ : no measurements, nor numerical viscosity used. This is the standard numerical solution of the wave equation using finite elements in space and a midpoint discretization scheme in time. We refer to the norm of the difference between this solution and the reference solution by the term "No observer error";

- $\gamma>0, \varepsilon=0$ : measurements are used without numerical viscosity. The norm of the difference between this solution and the reference solution is termed "Non-viscous observer error";

- $\gamma>0, \varepsilon=\max \{h, \Delta t\}$ : both measurements and numerical viscosity are used. The norm of the difference between this solution and the reference solution is called "Viscous observer error". 
All the error norms are plotted in relative values, namely, rescaled by dividing by the norm of the initial condition.

\subsection{One-dimensional wave equation}

Let $\Omega=(0,1)$ be the spatial domain in which we consider the one dimensional wave equation, and we take $\omega=(0.3,0.7)$ as the observation interval. The mesh underlying the $\mathbb{P}_{1}$-Lagrange finite element approximation subspace of $\mathcal{D}\left(A_{0}^{\frac{1}{2}}\right)=$ $H_{0}^{1}(0,1)$ is given by a uniform subdivision of the interval $(0,1)$ formed by $N$ internal points, and we denote $h=1 /(N+1)$. We choose the time step $\Delta t=0.8 h$, hence in the framework of Th. 2.1 we have $\varepsilon=h$. Note that we carefully keep away from the particular case $\Delta t=h$ to avoid specific phenomena such as super-convergence in the numerical assessments.

\subsubsection{Numerical assessments}

We performed some numerical experiments for various choices of initial conditions. In this case, the exact eigenmodes are the Fourier modes, hence we used the truncation of the modal decomposition as the reference solution.
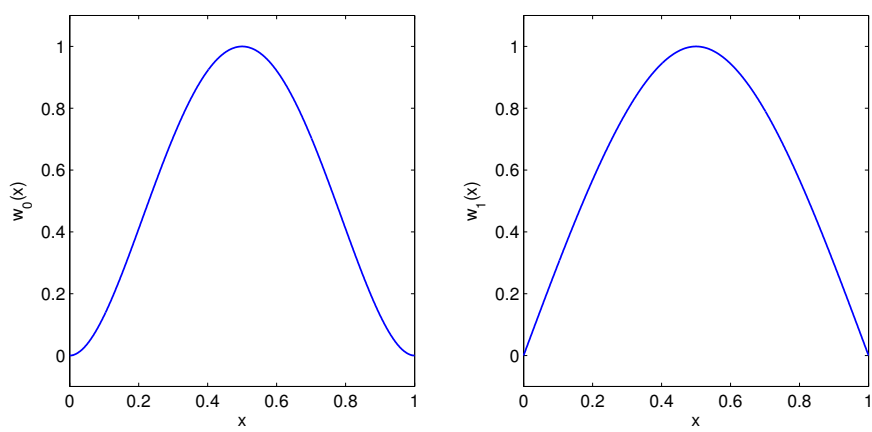

Fig. 1. Initial conditions in (5.7): $w_{0}$ (left) and $w_{1}$ (right)

Smooth initial data We consider the following initial conditions

$$
w_{0}(x)=16 x^{2}(1-x)^{2}, \quad w_{1}(x)= \begin{cases}3 x-4 x^{3}, & \text { if } x \in(0,0.5) \\ 4 x^{3}-12 x^{2}+9 x, & \text { if } x \in(0.5,1),\end{cases}
$$

displayed in Fig. 1, and which satisfy the regularity assumptions of Th. 2.1. The closed-form computation of the Fourier coefficients gives, $\forall k \in \mathbb{N}^{*}$,

$$
\left\{\begin{array}{l}
a_{k}=\frac{2 \sqrt{2}\left(\pi^{2} k^{2}-12\right)}{\pi^{5} k^{5}}\left((-1)^{k}-1\right) \\
b_{k}=\frac{48 \sqrt{2} \sin \frac{\pi k}{2}}{\pi^{4} k^{4}}
\end{array}\right.
$$




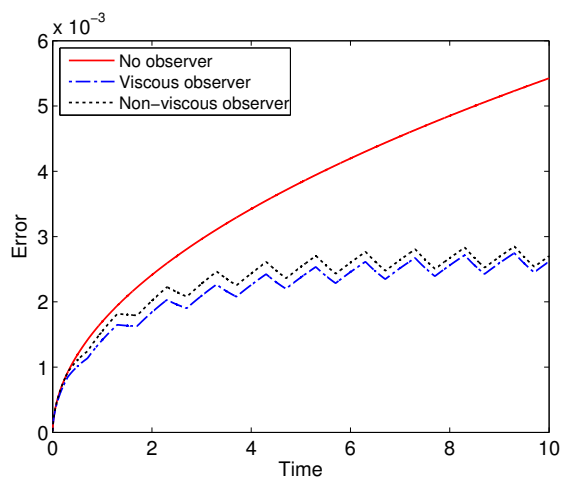

(a)

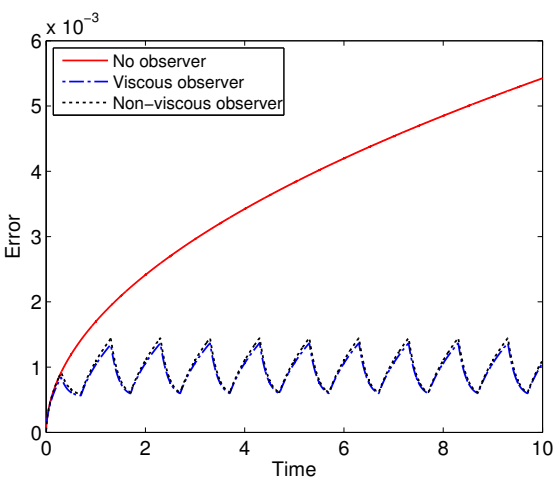

(b)

Fig. 2. Relative errors obtained for $N=1000$ and initial conditions (5.7) - Gain values: (a) $\gamma=1$; (b) $\gamma=9$

and we use $M=1000$ eigenmodes for the representation of the reference solution.

The relative errors obtained for the observer-based discretization (2.20) with $N=1000$ are displayed in Fig. 2 for the two gain values $\gamma \in\{1,9\}$. The numerical behavior observed is consistent with the above analysis, as the error for the viscous observer appears to reach an upper bound in time for the two gain values considered, whereas that of the standard discretization steadily deteriorates. Nevertheless, it is interesting to note that a very similar dramatic improvement is obtained with the observer-based discretization without added numerical viscosity. This will be further discussed in Section 5.1.2.
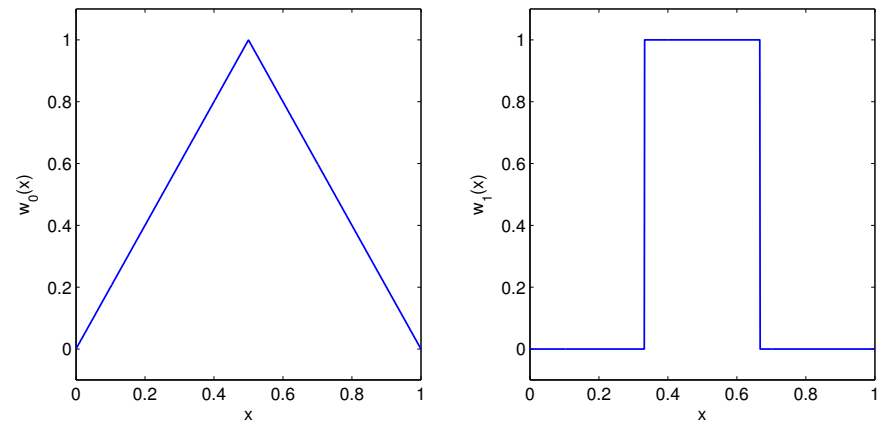

Fig. 3. Initial conditions in (5.8): $w_{0}$ (left) and $w_{1}$ (right) 
$\boldsymbol{H}_{\mathbf{0}}^{\mathbf{1}}$ initial displacement - discontinuous initial velocity We consider initial conditions defined by

$$
w_{0}(x)=1-|2 x-1|, \quad w_{1}(x)= \begin{cases}1, & \text { if } x \in\left(\frac{1}{3}, \frac{2}{3}\right) \\ 0, & \text { if not }\end{cases}
$$

and plotted in Fig. 3, which gives the following Fourier coefficients

$$
\left\{\begin{array}{l}
a_{k}=\frac{4 \sqrt{2}}{\pi^{2} k^{2}} \sin \frac{\pi k}{2} \\
b_{k}=\frac{1}{\pi k}\left(\cos \frac{\pi k}{3}-\cos \frac{2 \pi k}{3}\right)
\end{array}\right.
$$

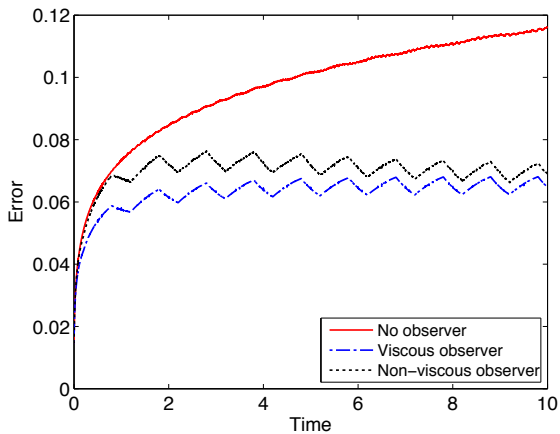

(a)

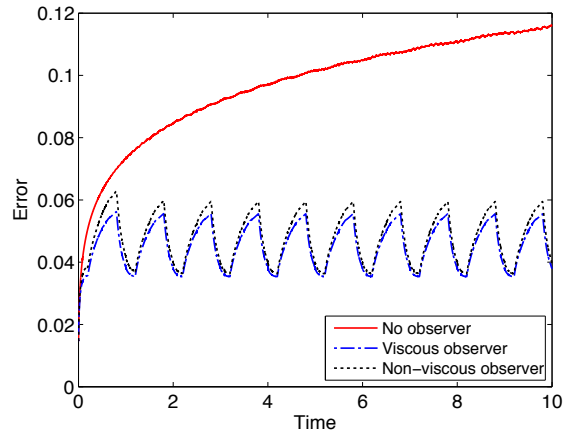

(b)

Fig. 4. Relative errors obtained for $N=1000$ and initial conditions (5.8) - Gain values: (a) $\gamma=1$; (b) $\gamma=9$

The corresponding relative errors are displayed in Fig. 4. In this case the initial conditions do not fulfill the regularity assumptions of Th. 2.1, and as a matter of fact all the computed errors are significantly larger than in the previous case. Nevertheless, all the other observations made for the results obtained with the smooth initial conditions also hold here.

\subsubsection{Pole locus analysis}

The location of the poles of the "closed-loop" operator $A-H^{*} H$ provides some valuable insight into the stability of the associated dynamical system, although of course the decay rate - in case of exponential stability - is only bounded by the opposite of the spectral abscissa (itself defined as the supremum of the real parts of the spectrum) for general infinite-dimensional systems. ${ }^{8,21}$ Note, however, that in some instances a complete stability analysis may be performed by analyzing the eigenpairs, see e.g. Ref. 7. Nevertheless, here we can compute the spectrum for the related discrete operators of interest, in which case the decay rate is exactly given 
by the opposite of the spectral abscissa. Hence, we will compute the eigenvalues (generically denoted by $\left(\lambda_{h}^{k}\right)_{k}$ ) of the following operators:

- $A_{h} \in \mathcal{L}\left(\mathcal{X}_{h}\right)$ which is the operator governing the discrete conservative system (3.1);

- $A_{h}-\gamma H_{h}^{*} H_{h}$ which governs the dynamics of a semi-discrete observer system without added viscosity;

- $A_{h}-\gamma H_{h}^{*} H_{h}+\varepsilon V_{\varepsilon}$ which governs the semi-discrete observer system with added viscosity, namely, the "theoretical" semi-discrete system associated with our proposed fully discrete scheme (2.20).

Moreover, since we are still more directly concerned with the fully discrete schemes themselves, we will compute the eigenvalues (generically denoted by $\left(\lambda_{h, \Delta t}^{k}\right)_{k}$ ) of the following corresponding operators:

- $\left(I-\frac{\Delta t}{2} A_{h}\right)^{-1}\left(I+\frac{\Delta t}{2} A_{h}\right)$;

- $\left(I-\frac{\Delta t}{2} A_{h}+\gamma \frac{\Delta t}{2} H_{h}^{*} H_{h}\right)^{-1}\left(I+\frac{\Delta t}{2} A_{h}-\gamma \frac{\Delta t}{2} H_{h}^{*} H_{h}\right)$;

- $\left(I-\frac{\Delta t}{2} A_{h}+\gamma \frac{\Delta t}{2} H_{h}^{*} H_{h}-\Delta t \varepsilon V_{\varepsilon}+\frac{\Delta t^{2}}{2} \varepsilon A_{h} V_{\varepsilon}+\gamma \frac{\Delta t^{2}}{2} \varepsilon H_{h}^{*} H_{h} V_{\varepsilon}\right)^{-1}\left(I+\frac{\Delta t}{2} A_{h}-\right.$ $\left.\gamma \frac{\Delta t}{2} H_{h}^{*} H_{h}\right)$.

In the totally discrete case we will represent the post-processed values given by $\frac{\log \left(\lambda_{h, \Delta t}^{k}\right)}{\Delta t}$ to allow a direct comparison with the eigenvalues of the corresponding semi-discrete operators.
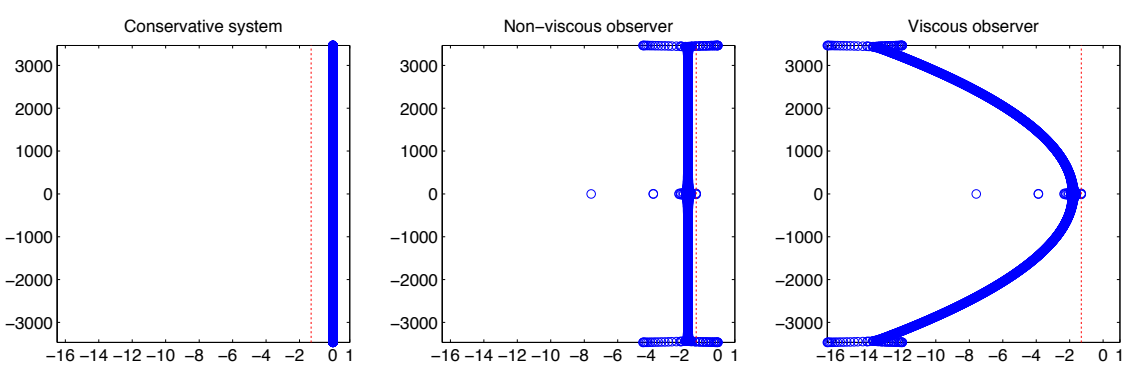

Fig. 5. Eigenvalues for space semi-discrete systems

We display in Figures 5 and 6 the pole loci of the semi-discrete and fully discrete operators, respectively. For both figures we considered $N=1000$ discretization points, $\omega=(0.3,0.7)$ and $\gamma=9$. We observe similar trends in both cases, namely,

- the closed-loop observer term $-\gamma H_{h}^{*} H_{h}$ provides some damping by itself; however, this is not uniform for all eigenvalues, and some high-frequency poles have "vanishing" real parts;

- this difficulty is effectively tackled by the added viscosity operator, which ensures uniform damping, i.e. we observe the same effect on the numerical poles as 

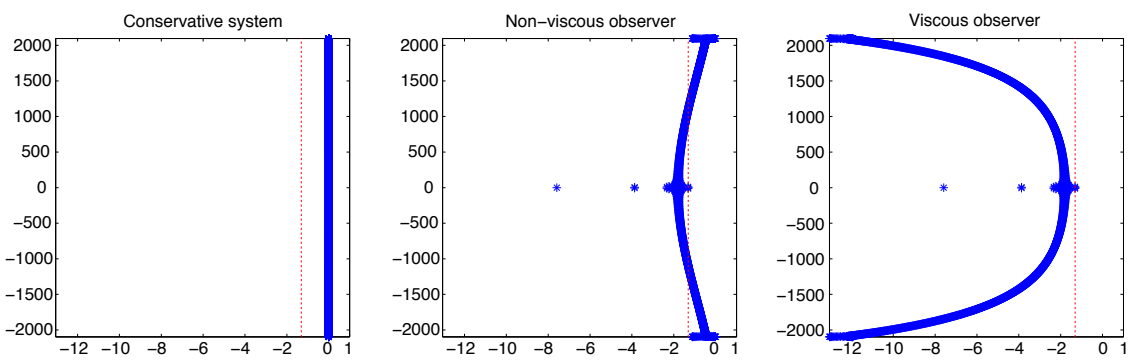

Fig. 6. Post-processed eigenvalues for fully discrete systems

e.g. in Ref. 12 which considers the semi-discrete problem for a $1 \mathrm{D}$ wave equation stabilized in both equations of the equivalent first-order system written for the gradient and time-derivative of $w$.

This in fact provides an interpretation of the limited effect of the added numerical viscosity in our above numerical assessments, as the modal components corresponding to the weakly-damped high-frequency poles are likely to be very small due to the relative regularity of the initial conditions.

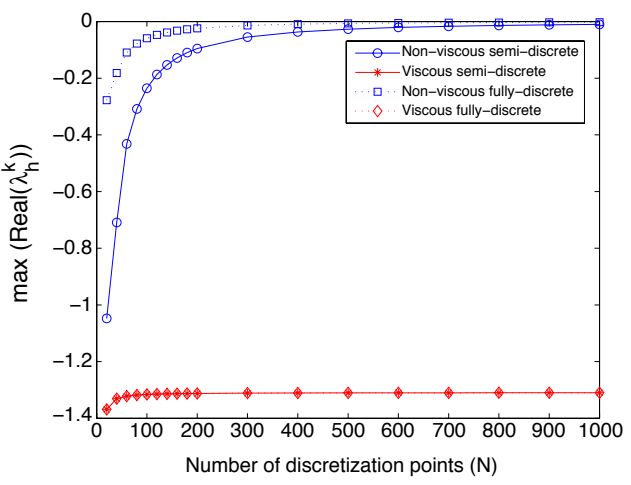

Fig. 7. Maximum of $\operatorname{Re}\left(\lambda_{k}^{h}\right)$ (semi-discrete) and $\operatorname{Re} \log \left(\lambda_{h, \Delta t}^{k}\right) / \Delta t$ (fully discrete) when varying $N-$ Gain value $\gamma=9$

It is also interesting to analyze the variations of these eigenvalues with respect to various parameters. To that effect we first investigate in Fig. 7 the impact of the spatial discretization parameter by displaying the spectral abscissa as a function of the subdivision number $N$. We observe that without viscosity operator this indicator deteriorates for both semi-discrete and totally discrete cases when refining the discretization, as expected from Figures 5 and 6 . By contrast, a very uniform behavior is obtained when numerical viscosity is present. 


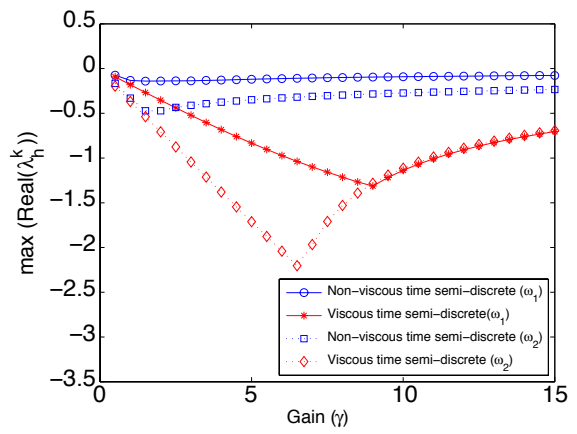

(a)

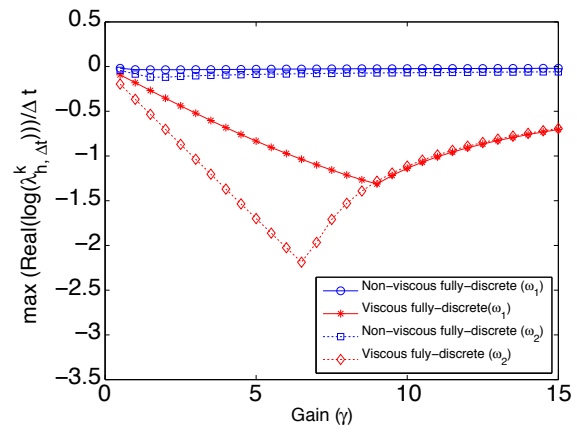

(b)

Fig. 8. Effect of gain parameter and observation set: (a) Max. of $\operatorname{Re}\left(\lambda_{k}^{h}\right)-$ (b) Max. of $\operatorname{Re} \log \left(\lambda_{h, \Delta t}^{k}\right) / \Delta t$

Finally, we study the issue of the $\gamma$ gain parameter adjustment for a given observation set. In Fig. 8 we plot the spectral abscissa for varying values of $\gamma$ and for two observation sets $\omega_{1}=(0.3,0.7)$ and $\omega_{2}=(0.1,0.8)$. As expected, the optimal spectral abscissa is improved for a larger observation range. Moreover, the value $\gamma=1$ is clearly too small to provide effective damping whereas $\gamma=9$ is close to the optimal value for $\omega_{1}$, and this explains the differences observed for these two values in Figures 2 and 4 . Nevertheless, while optimal gain parameter values can be identified for each observation set and over-damping tends to occur for larger gain values, in the presence of numerical viscosity the system is effectively stabilized for a wide range of gain values. This behavior - together with the already mentioned stability with respect to the discretization parameter - allows to easily calibrate the gain parameter to achieve best performance.

\subsection{Two-dimensional wave equation in a square}

Let $\Omega=(0,1)^{2}$ be the unit square, for which we can easily compute the eigenfunctions of the Laplacian, namely, the tensor products of the above Fourier modes

$$
\phi_{k l}=\sin (k \pi x) \sin (l \pi y), \quad k, l \in \mathbb{N}^{*} .
$$

An interesting feature of this domain is that - unlike in the $1 \mathrm{D}$ case and more "arbitrary" 2D shapes - we can devise a measurement set that does not satisfy the geometric control condition of Ref. 2 . In particular, in addition to

$$
\omega_{1}=\left\{(0,1) \times\left(\left(0, \frac{1}{10}\right) \cup\left(\frac{9}{10}, 1\right)\right)\right\} \cup\left\{\left(\left(0, \frac{1}{10}\right) \cup\left(\frac{9}{10}, 1\right)\right) \times(0,1)\right\},
$$

which fulfills the condition, we will consider

$$
\omega_{2}=\left(0, \frac{1}{10}\right) \times(0,1)
$$

which does not. 
The triangular meshes are obtained by subdividing the domain into $(N-1)^{2}$ square elements, and splitting each resulting square into two triangles, hence we have a uniform element diameter $h=1 /(N-1)$. We here take $\Delta t=1.2 h$. In practice, in our numerical assessments we will take $N=50$, compute the reference solution using $50^{2}$ analytically computed eigenmodes, and set the observer gain to $\gamma=6$ based on a preliminary calibration performed with the poles.
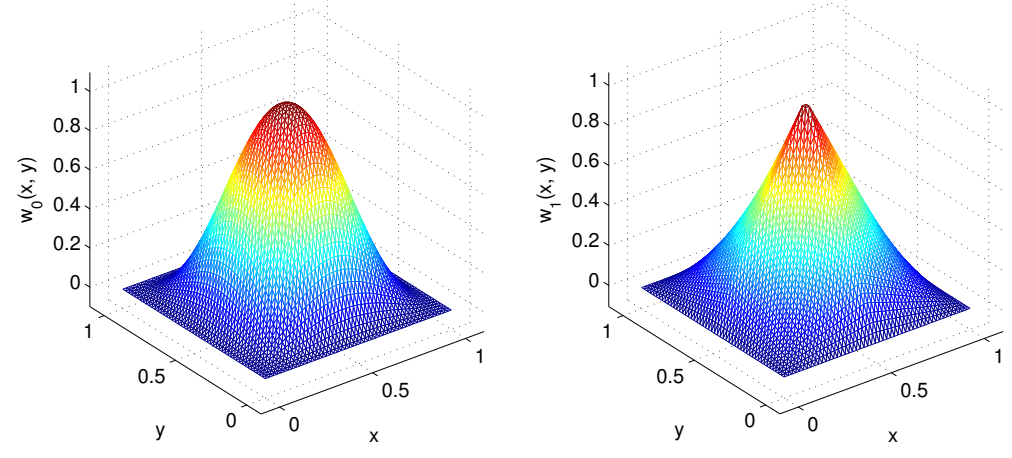

Fig. 9. Initial conditions in (5.9)

Smooth initial displacement $-\boldsymbol{H}_{0}^{1}$ initial velocity We first consider the initial conditions given by

$$
w_{0}(x, y)=256 x^{2}(1-x)^{2} y^{2}(1-y)^{2}, \quad w_{1}(x, y)=(1-|2 x-1|)(1-|2 y-1|),
$$

see Fig. 9, with associated modal coefficients

$$
\left\{\begin{array}{l}
a_{k l}=\frac{32 \sqrt{2}}{\pi^{5}\left(k^{5}+l^{5}\right)}\left(\left(\pi^{2}\left(k^{2}+l^{2}\right)-12\right)\left(\cos \left(\pi \sqrt{k^{2}+l^{2}}\right)-1\right)\right. \\
\left.\quad-12 \pi \sqrt{k^{2}+l^{2}} \sin \left(\pi \sqrt{k^{2}+l^{2}}\right)\right), \\
b_{k l}=\frac{4 \sqrt{2}}{\pi \sqrt{k^{2}+l^{2}}}\left(2 \sin \frac{\pi \sqrt{k^{2}+l^{2}}}{2}-\sin \left(\pi \sqrt{k^{2}+l^{2}}\right)\right),
\end{array}\right.
$$

The errors obtained in this case are plotted in Fig. 10. When the measurement domain satisfies the observability condition the resulting behavior is very similar to the 1D case. By contrast, without the observability condition the errors associated with observer discretizations do not appear to be bounded in time, albeit still grow more slowly than for the standard discretization.

$H_{0}^{1}(\Omega)$ initial displacement - discontinuous velocity We then consider the less regular initial conditions

$$
w_{0}(x, y)=(1-|2 x-1|)(1-|2 y-1|), \quad w_{1}(x, y)=\chi_{\left(\frac{1}{3}, \frac{2}{3}\right)^{2}}(x, y),
$$




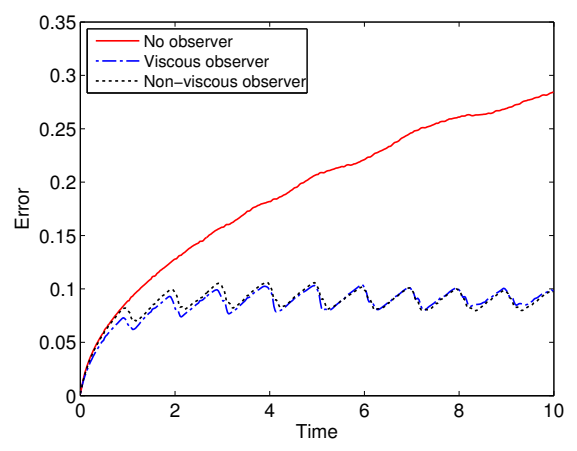

(a)

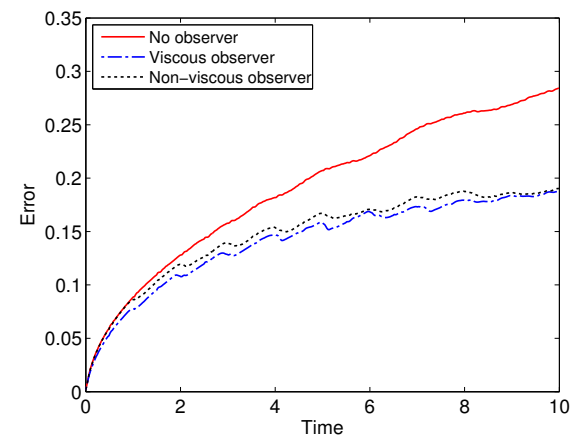

(b)

Fig. 10. Relative errors corresponding to initial conditions (5.9) for measurement sets $\omega=\omega_{1}$ (a) and $\omega=\omega_{2}(\mathrm{~b})$
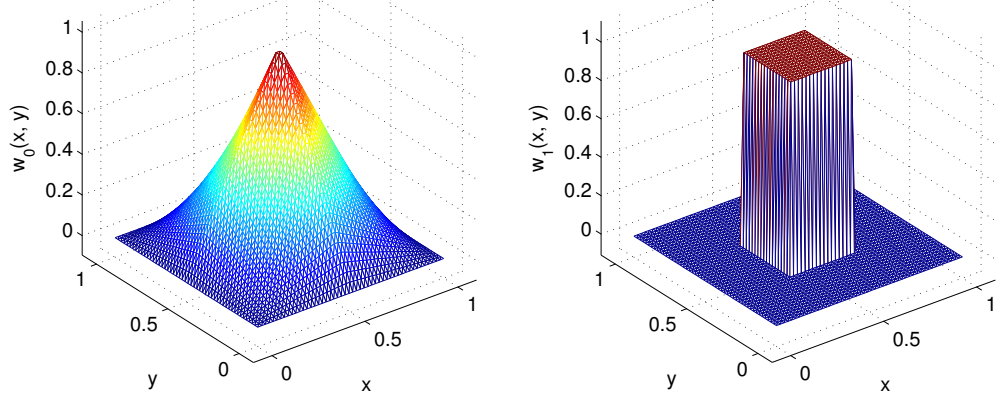

Fig. 11. Initial conditions in (5.10)

where $\chi_{\mathcal{O}}$ denotes the characteristic function of the set $\mathcal{O} \subset \Omega$, see Fig. 11. The corresponding modal coefficients are

$$
\left\{\begin{array}{l}
a_{k l}=\frac{4 \sqrt{2}}{\pi \sqrt{k^{2}+l^{2}}}\left(2 \sin \frac{\pi \sqrt{k^{2}+l^{2}}}{2}-\sin \left(\pi \sqrt{k^{2}+l^{2}}\right)\right) \\
b_{k l}=\frac{\sqrt{2}}{\pi \sqrt{k^{2}+l^{2}}}\left(\cos \frac{\pi \sqrt{k^{2}+l^{2}}}{3}-\cos \frac{2 \pi \sqrt{k^{2}+l^{2}}}{3}\right)
\end{array}\right.
$$

In the resulting errors plotted in Fig. 12, the main differences with the previous smoother case is that the error values are larger - as expected - and the performance of the viscous observer is now more clearly distinguished. A natural interpretation of this fact is that high-frequency poles are much more present here due to the reduced regularity in the initial condition. 


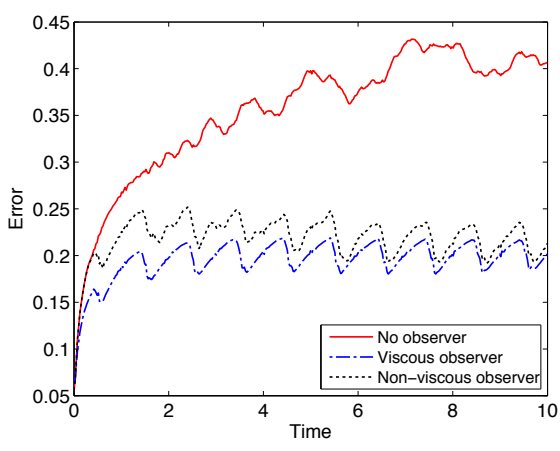

(a)

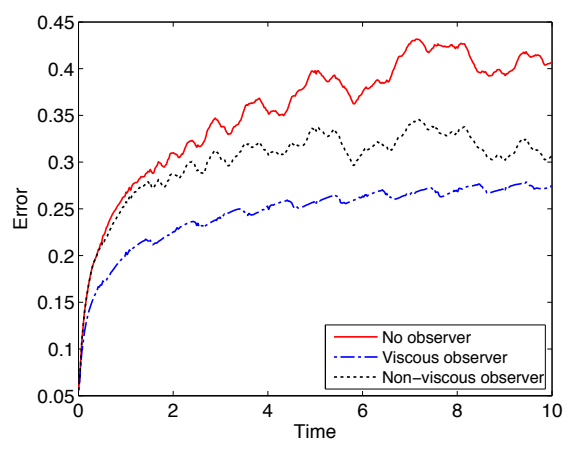

(b)

Fig. 12. Relative errors corresponding to initial conditions (5.10) for measurement sets $\omega=\omega_{1}$ (a) and $\omega=\omega_{2}(\mathrm{~b})$

\subsection{Two-dimensional wave equation in an arbitrary domain}

In this section we demonstrate some applications of our proposed method to the twodimensional wave equation in a spatial domain of more general shape, see Fig. 13.

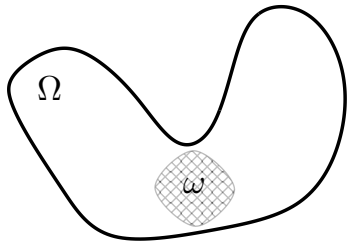

(a)

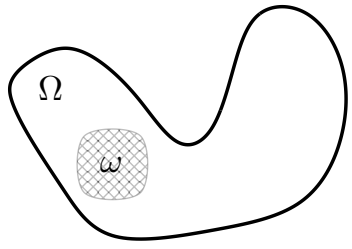

(b)

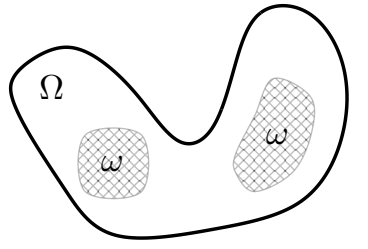

(c)

Fig. 13. Domain and observation sets for two-dimensional wave equation

Of course, unlike in the previous cases here we cannot compute the eigenfunctions or the eigenvalues of the operator $A_{0}$ analytically, and therefore we need to resort to approximate reference solutions. To that purpose we will consider two categories of meshes for the domain $\Omega$ : target computational meshes for which we want to assess our numerical strategy, and substantially more refined meshes to compute the numerical reference solution. In order to have conformity of the meshes between the measurement subset and the rest of the domain, we thus define one pair of triangular meshes for each $\omega$ considered, see Tab. 5.3 listing the number of nodes in $\Omega$ in each case corresponding to Fig. 13 .

We consider the initial displacement displayed in Fig. 14 - given by a static response to a uniform surface-distributed loading - and zero initial velocity. The time step is $\Delta t=0.01$, and based on a spectral calibration we choose $\gamma=3$. The numerical errors - in relative norm values - with respect to the reference solutions 
Table 1. Number of nodes in the meshes corresponding to geometries in Fig. 13

\begin{tabular}{cccc}
\hline Number of nodes & (a) & (b) & (c) \\
\hline Refined mesh & 3694 & 3730 & 3703 \\
Target mesh & 948 & 958 & 949 \\
\hline
\end{tabular}

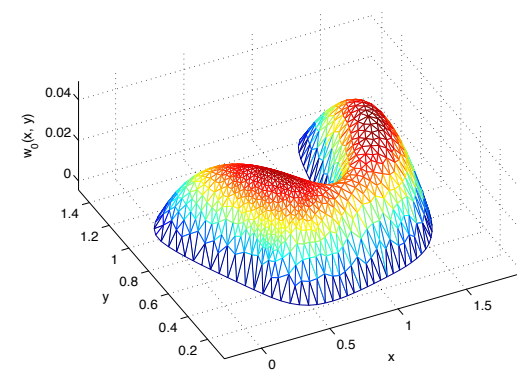

Fig. 14. Initial displacement for the wave equation

computed with the refined meshes are shown in Fig. 15, for each observation set displayed in Fig. 13. It is very interesting to correlate the measured accuracy with the "degree of observability" associated with each case, as here while observability always holds in the strict sense the observability constant can be expected to vary. In case (a) the measurement set is rather limited in extent, but centrally located, and indeed the observer then performs slightly better than in case (b). Then, when incorporating an additional measurement patch in case (c) we obtain a dramatically improved accuracy, in what can be naturally interpreted as a configuration of rather high observability - despite the fact that the geometric control condition is not satisfied due to an unobserved ray near the center of the domain. In this last case we have a simulation error consistent with what would be the interpolation error for the type of - rather coarse, see Fig. 14 - mesh considered, albeit stable over time.

\section{Application to beam and plate models}

In this section we now adapt the method developed in this paper to the case where the governing operator $A_{0}$ is fourth-order in space. More specifically, let $\Omega \subset \mathbb{R}^{n}$ be an open and non-empty set with Lipschitz boundary and consider the following equations

$$
\begin{cases}\ddot{w}(t, x)+\Delta^{2} w(t, x)=0, & (t, x) \in(0, T) \times \Omega \\ w(t, x)=\Delta w(t, x)=0, & (t, x) \in(0, T) \times \partial \Omega \\ w(0, x)=w_{0}(x), \quad \dot{w}(0, x)=w_{1}(x), & x \in \Omega .\end{cases}
$$




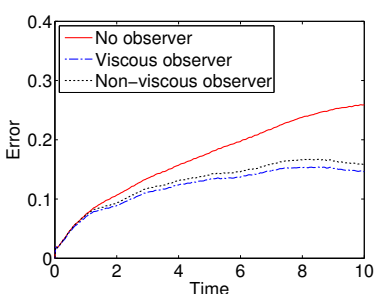

(a)

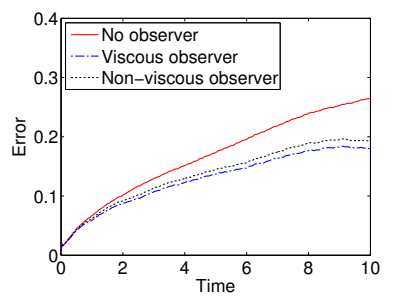

(b)

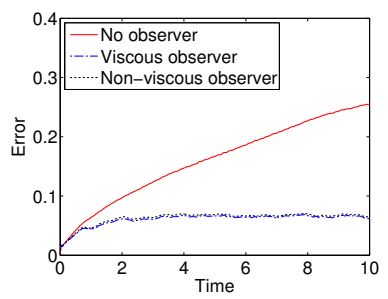

(c)

Fig. 15. Error between the reference solution and the solution computed on the low resolution mesh, for each observation set displayed in Fig. 13.

This model covers the cases of the Euler-Navier-Bernoulli beam model in dimension 1 , and of the Kirchhoff-Love plate model in dimension 2, both for so-called simplysupported boundary conditions, and in these cases $w$ represents the deflection of the structure, see e.g. Ref. 4.

\subsection{Mathematical setting}

We denote $\mathcal{H}=L^{2}(\Omega)$, and $A_{0}: \mathcal{D}\left(A_{0}\right) \rightarrow \mathcal{H}$ is defined by

$$
\mathcal{D}\left(A_{0}\right)=\left\{\varphi \in H^{4}(\Omega) \mid \varphi=\Delta \varphi=0 \text { on } \partial \Omega\right\}, \quad A_{0} \varphi=\Delta^{2} \varphi \text { for all } \varphi \in \mathcal{D}\left(A_{0}\right) .
$$

It is easy to verify that $A_{0}$ is a self-adjoint positive definite operator with compact resolvent, and $A_{0}^{\frac{1}{2}}: \mathcal{D}\left(A_{0}^{\frac{1}{2}}\right) \rightarrow \mathcal{H}$ is given by

$$
\mathcal{D}\left(A_{0}^{\frac{1}{2}}\right)=H^{2}(\Omega) \cap H_{0}^{1}(\Omega), \quad A_{0}^{\frac{1}{2}} \varphi=-\Delta \varphi \text { for all } \varphi \in \mathcal{D}\left(A_{0}^{\frac{1}{2}}\right) .
$$

Accordingly, with measurements given by

$$
z(t)=\left.w(\cdot, t)\right|_{\omega} ; \quad t \geq 0
$$

we need to select a lifting operator $\mathcal{L}_{\omega}$ compatible with the required regularities, hence we define $\mathcal{L}_{\omega}: \mathcal{Z}=H^{2}(\omega) \rightarrow \mathcal{D}\left(A_{0}^{\frac{1}{2}}\right)=H^{2}(\Omega) \times H_{0}^{1}(\Omega)$ by $\mathcal{L}_{\omega} \phi=\psi$ with

$$
\begin{cases}\Delta^{2} \psi=0, & \text { in } \Omega \backslash \omega \\ \psi=\Delta \psi=0, & \text { on } \partial \Omega \\ \frac{\partial \psi}{\partial n}=\frac{\partial \phi}{\partial n}, & \text { on } \partial \omega \backslash \partial \Omega \\ \psi=\phi, & \text { in } \bar{\omega}\end{cases}
$$

We equip $\mathcal{Z}=H^{2}(\omega)$ with the inner product

$$
\langle\phi, \psi\rangle_{H_{\omega}^{2}}=\left\langle\Delta \mathcal{L}_{\omega} \phi, \Delta \mathcal{L}_{\omega} \psi\right\rangle_{L^{2}(\Omega)}, \quad \phi, \psi \in H^{2}(\omega),
$$

and it is quite straightforward to see that the associated norm is equivalent to the usual norm of $H^{2}(\omega)$, under the standard regularity assumptions on the domain 
boundary. Let now $H_{0} \in \mathcal{L}\left(\mathcal{D}\left(A_{0}^{\frac{1}{2}}\right), \mathcal{Z}\right)$ be the observation operator defined according to (6.2), namely,

$$
H_{0} \phi=\left.\phi\right|_{\omega}
$$

It is easy to check that $H_{0}^{*} \in \mathcal{L}\left(\mathcal{Z}, \mathcal{D}\left(A_{0}^{\frac{1}{2}}\right)\right)$ is nothing else than $\mathcal{L}_{\omega}$.

In this case, we can also prove an observability inequality in the form (2.3), see Prop. Appendix A.1 in Appendix. Therefore we can consider the discretization scheme (2.20) and Th. 2.1 applies.

\subsection{Beam equation and numerical assessment}

We now particularize System (6.1) to the one-dimensional setting, namely, corresponding to the beam model. We thus consider

$$
\begin{cases}\ddot{w}(t, x)+w_{, x x x x}(t, x)=0, & (t, x) \in(0, T) \times(0,1) \\ w(t, 0)=w_{, x x}(t, 0)=0, & t \in(0, T) \\ w(t, 1)=w_{, x x}(t, 1)=0, & t \in(0, T) \\ w(0, x)=w_{0}(x), \quad \dot{w}(0, x)=w_{1}(x), & x \in(0,1)\end{cases}
$$

with a finite element discretization obtained with $\mathbb{P}_{3}$-Hermite elements in a uniform subdivision of the interval $(0,1)$ formed by $N$ internal points, and we denote $h=1 /(N+1)$. The corresponding projectors $\pi_{h}$ and $\check{\pi}_{h}$ then satisfy all the required assumptions (2.6)-(2.11) with $\theta=2$, see e.g. Ref. 36 (p.144). For the time discretization we take $\Delta t=1.28 h^{2}$, and still $\varepsilon=\max \left\{h^{2}, \Delta t\right\}$ in the numerical viscosity.

In our numerical assessment we set $N=100, \omega=(0.3,0.7)$ and we consider the initial condition $\left(\begin{array}{c}w_{0} \\ w_{1}\end{array}\right) \in \mathcal{D}\left(A_{0}^{\frac{3}{2}}\right) \times \mathcal{D}\left(A_{0}\right)$ given by

$$
w_{0}(x)=\alpha x^{7}(1-x)^{5}, \quad w_{1}(x)=0, \quad x \in(0,1),
$$

where $\alpha$ is a scaling constant such that $\left\|w_{0}\right\|_{\mathcal{D}\left(A_{0}^{\frac{1}{2}}\right)}=1$. Here again, the exact eigenmodes are the Fourier modes and we can compute the modal coefficients for the above initial conditions to provide a reference solution of arbitrary accuracy, given here for completeness, viz.

$$
\begin{aligned}
& a_{k}=-120 \alpha \frac{42 \pi^{6} k^{6}-280 \pi^{4} k^{4}+15120 \pi^{2} k^{2}-95040}{\pi^{13} k^{13}}(-1)^{k} \\
&+1680 \alpha \frac{\pi^{4} k^{4}-180 \pi^{2} k^{2}+2376}{\pi^{13} k^{13}} \sqrt{2} .
\end{aligned}
$$

The assessment results are shown in Fig. 16. Here again, the effectiveness of our approach is fully confirmed in the numerical results, while the very limited effect of numerical viscosity can be attributed to the regularity of the initial condition. 


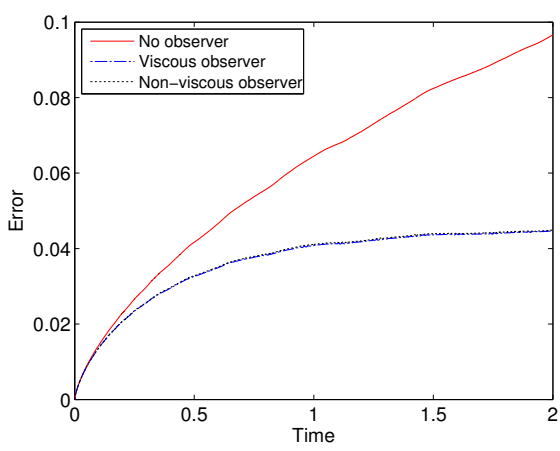

(a)

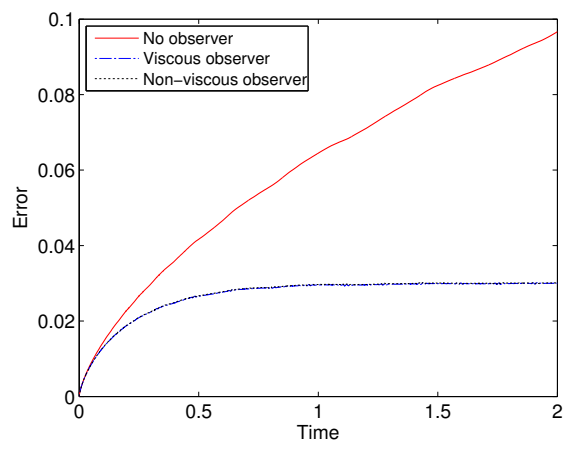

(b)

Fig. 16. Relative errors for beam equation with $N=100$ and initial condition (6.7) - Gain values: (a) $\gamma=5$; (b) $\gamma=10$.

\section{Appendix A. An observability inequality for the space-wise fourth-order equation}

In this appendix, we establish the required continuous observability condition for the space-wise fourth-order equation - without any assumption on the space dimension. Let $\check{\omega} \subset \Omega$ be an open and non-empty subset of $\Omega$. It is well-known that if $\check{\omega}$ satisfies the geometric control condition of Ref. 2 the following observability inequality holds for the solution of $(6.1)$,

$$
\int_{0}^{\check{T}} \int_{\check{\omega}}|\dot{w}(t, x)|^{2} \mathrm{~d} x \mathrm{~d} t \geq k_{T}\left(\left\|w_{0}\right\|_{\mathcal{D}\left(A_{0}^{\frac{1}{2}}\right)}^{2}+\left\|w_{1}\right\|_{\mathcal{H}}^{2}\right),
$$

for any $\check{T}>0$, see e.g. Ref. 20. The aim of this appendix is to prove an alternative observability inequality for our observation operator $H_{0}$ in (6.5).

Proposition Appendix A.1. Assume that $\check{\omega} \subset \Omega$ and $\check{T}>0$ are such that the inequality (A.1) holds. Then, for every open set $\omega \in \Omega$ with $\check{\omega} \subsetneq \omega$ and $\operatorname{dist}(\Omega \backslash$ $\omega, \check{\omega})>0$ and for every $T>\check{T}$ the following observability inequality holds

$$
\int_{0}^{T}\|w(t, \cdot)\|_{H^{2}(\omega)}^{2} d t \geq k_{T}^{2}\left(\left\|w_{0}\right\|_{\mathcal{D}\left(A_{0}^{\frac{1}{2}}\right)}^{2}+\left\|w_{1}\right\|_{H}^{2}\right) .
$$

Proof. The proof is quite similar to that performed in Prop. 2.2 of Ref. 5 for the wave equation, hence we only outline the main steps for completeness. Let $\psi \in C_{c}^{\infty}(\bar{\Omega})$ be a smooth function with values between 0 and 1 and such that

$$
\psi(x)= \begin{cases}0, & x \in \Omega \backslash \omega \\ 1, & x \in \check{\omega}\end{cases}
$$

and $\phi(t)=t^{2}(\check{T}-t)^{2}$. Multiplying the first equation in (6.1) by $\psi \phi w$ and integrating 
by parts, we obtain

$$
\begin{aligned}
0= & \int_{0}^{\check{T}} \int_{\omega} \phi \psi\left(\ddot{w}+\Delta^{2} w\right) w \mathrm{~d} x \mathrm{~d} t \\
= & \int_{0}^{\check{T}} \int_{\omega} \ddot{\varphi} \psi \frac{|w|^{2}}{2} \mathrm{~d} x \mathrm{~d} t-\int_{0}^{\check{T}} \int_{\omega} \phi \psi|\dot{w}|^{2} \mathrm{~d} x \mathrm{~d} t \\
& +\int_{0}^{\check{T}} \int_{\partial \omega} \phi \psi w \frac{\partial}{\partial n}(\Delta w) \mathrm{d} \sigma(x) \mathrm{d} t-\int_{0}^{\check{T}} \int_{\omega} \phi \nabla(\psi w) \cdot \nabla(\Delta w) \mathrm{d} x \mathrm{~d} t .
\end{aligned}
$$

The third term in the right-hand side vanishes because $\left.\psi\right|_{\partial \omega}=0$. Performing some further manipulations on the last term we obtain

$$
\begin{aligned}
-\int_{0}^{\check{T}} & \int_{\omega} \phi \nabla(\psi w) \cdot \nabla(\Delta w) \mathrm{d} x \mathrm{~d} t \\
\quad= & \int_{0}^{\check{T}} \int_{\omega} \phi \Delta(\psi w) \Delta w \mathrm{~d} x \mathrm{~d} t-\int_{0}^{\check{T}} \int_{\partial \omega} \phi \frac{\partial}{\partial n}(\psi w) \Delta w \mathrm{~d} \sigma(x) \mathrm{d} t \\
= & \int_{0}^{\check{T}} \int_{\omega}(\phi \Delta \psi) w \Delta w \mathrm{~d} x \mathrm{~d} t+\int_{0}^{\check{T}} \int_{\omega} \phi \psi|\Delta w|^{2} \mathrm{~d} x \mathrm{~d} t \\
& +\int_{0}^{\check{T}} \int_{\omega} 2 \phi(\nabla \psi \cdot \nabla w) \Delta w \mathrm{~d} x \mathrm{~d} t .
\end{aligned}
$$

Substituting into (A.3) we get

$$
\begin{aligned}
\int_{0}^{\check{T}} \int_{\omega} \phi \psi|\dot{w}|^{2} \mathrm{~d} x & \mathrm{~d} t=\int_{0}^{\check{T}} \int_{\omega} \phi \psi|\Delta w|^{2} \mathrm{~d} x \mathrm{~d} t+\int_{0}^{\check{T}} \int_{\omega} \ddot{\varphi} \psi \frac{|w|^{2}}{2} \mathrm{~d} x \mathrm{~d} t \\
& +\int_{0}^{\check{T}} \int_{\omega}(\phi \Delta \psi) w \Delta w \mathrm{~d} x \mathrm{~d} t+\int_{0}^{\check{T}} \int_{\omega} 2 \phi(\nabla \psi \cdot \nabla w) \Delta w \mathrm{~d} x \mathrm{~d} t
\end{aligned}
$$

Since all the derivatives of $w$ appearing in the right-hand side are only up to secondorder, and given the positiveness of the cutoff functions $\phi$ and $\psi$, we infer that for any $\varepsilon>0$ we have

$$
C_{\varepsilon} \int_{\varepsilon}^{\check{T}-\varepsilon} \int_{\check{\omega}}|\dot{w}(t, x)|^{2} \mathrm{~d} x \mathrm{~d} t \leq \int_{0}^{\check{T}}\|w(t, \cdot)\|_{H^{2}(\omega)}^{2} \mathrm{~d} t,
$$

and we conclude the proof like in Ref. 5 by time-shifting the solution of (6.1).

\section{References}

1. D. Auroux and J. Blum. A nudging-based data assimilation method: the Back and Forth Nudging (BFN) algorithm. Nonlinear Processes Geophysics, 15:305-319, 2008.

2. C. Bardos, G. Lebeau, and J. Rauch. Sharp sufficient conditions for the observation, control, and stabilization of waves from the boundary. SIAM J. Control Optim., 30(5):1024-1065, 1992.

3. S.C. Brenner and L.R. Scott. The Mathematical Theory of Finite Element Methods, volume 15 of Texts in Applied Mathematics. Springer-Verlag, New York, 1994. 
4. D. Chapelle and K.J. Bathe. The Finite Element Analysis of Shells - Fundamentals. Springer, second edition, 2011.

5. D. Chapelle, N. Cîndea, M. de Buhan, and P. Moireau. Exponential convergence of an observer based on partial field measurements for the wave equation. Research Report 7728, INRIA, 2011. http://hal.inria.fr/inria-00619504/fr.

6. D. Chapelle, P. Moireau, and P. Le Tallec. Robust filtering for joint stateparameter estimation in distributed mechanical systems. DCDS-A, 23(1-2):65-84, 2009. doi:10.3934/dcds.2009.23.65.

7. S. Cox and E. Zuazua. The rate at which energy decays in a damped string. Comm. Partial Differential Equations, 19(1-2):213-243, 1994.

8. R.F. Curtain and H. Zwart. An Introduction to Infinite-Dimensional Linear Systems Theory, volume 21 of Texts in Applied Mathematics. Springer-Verlag, New York, 1995.

9. R. Dautray and J.-L. Lions. Mathematical Analysis and Numerical Methods for Science and Technology. Vol. 5. Springer-Verlag, Berlin, 1992.

10. S. Ervedoza. Spectral conditions for admissibility and observability of wave systems: applications to finite element schemes. Numer. Math., 113(3):377-415, 2009.

11. S. Ervedoza, C. Zheng, and E. Zuazua. On the observability of time-discrete conservative linear systems. Journal of Functional Analysis, 254(12):3037-3078, 2008.

12. S. Ervedoza and E. Zuazua. Perfectly matched layers in 1-d: energy decay for continuous and semi-discrete waves. Numer. Math., 109(4):597-634, 2008.

13. S. Ervedoza and E. Zuazua. Uniformly exponentially stable approximations for a class of damped systems. J. Math. Pures Appl. (9), 91(1):20-48, 2009.

14. P. Grisvard. Elliptic Problems in Nonsmooth Domains, volume 24 of Monographs and Studies in Mathematics. Pitman (Advanced Publishing Program), Boston, MA, 1985.

15. B.Z. Guo and Z.C. Shao. Stabilization of an abstract second order system with application to wave equations under non-collocated control and observations. Systems 86 Control Letters, 58(5):334-341, 2009.

16. G. Haine and K. Ramdani. Reconstructing initial data using observers. Numer Math, pages 1-37, August 2011.

17. A. Haraux. Une remarque sur la stabilisation de certains systèmes du deuxième ordre en temps. Portugaliae Mathematica, 46(3):245-258, 1989.

18. M. Krstic, B.-Z. Guo, A. Balogh, and A. Smyshlyaev. Control of a Tip-Force Destabilized Shear Beam by Observer-Based Boundary Feedback. SIAM Journal on Control and Optimization, 47(2):553, 2008.

19. I. Lasiecka and R. Triggiani. $L_{2}(\Sigma)$-regularity of the boundary to boundary operator $B^{*} L$ for hyperbolic and Petrowski PDEs. Abstr. Appl. Anal., (19):1061-1139, 2003.

20. G. Lebeau. Contrôle de l'équation de Schrödinger. J. Math. Pures Appl. (9), 71(3):267291, 1992.

21. G. Lebeau. Equation des ondes amorties. In Algebraic and geometric methods in mathematical physics, volume 19 of Math. Phys. Stud., pages 73-109. Kluwer Academic, 1996.

22. X.-D. Li and C.-Z. Xu. Infinite-dimensional Luenberger-like observers for a rotating body-beam system. Systems \& Control Letters, 60(2):138-145, 2011.

23. K. Liu. Locally distributed control and damping for the conservative systems. SIAM J. Control Optim., 35(5):1574-1590, 1997.

24. D.G. Luenberger. An introduction to observers. IEEE Transactions on Automatic Control, 16:596-602, 1971.

25. L. Miller. Controllability cost of conservative systems: resolvent condition and transmutation. J. Funct. Anal., 218(2):425-444, 2005.

26. L. Miller. Resolvent conditions for the control of unitary groups and their approxima- 
tions. Hal archives ouvertes, 2011.

27. P. Moireau and D. Chapelle. Reduced-order Unscented Kalman Filtering with application to parameter identification in large-dimensional systems. COCV, 17:380-405, 2011. doi: $10.1051 / \mathrm{cocv} / 2010006$.

28. P. Moireau, D. Chapelle, and P. Le Tallec. Joint state and parameter estimation for distributed mechanical systems. Computer Methods in Applied Mechanics and Engineering, 197:659-677, 2008.

29. P. Moireau, D. Chapelle, and P. Le Tallec. Filtering for distributed mechanical systems using position measurements: Perspectives in medical imaging. Inverse Problems, 25(3):035010 (25pp), 2009. doi:10.1088/0266-5611/25/3/035010.

30. P. Moireau, D. Chapelle, and P. Le Tallec. Filtering for distributed mechanical systems using position measurements: perspectives in medical imaging. Inverse Problems, 25(3):035010, 25, 2009.

31. A. Pazy. Semigroups of Linear Operators and Applications to Partial Differential Equations, volume 44 of Applied Mathematical Sciences. Springer-Verlag, New York, 1983.

32. K. Ramdani, T. Takahashi, G. Tenenbaum, and M. Tucsnak. A spectral approach for the exact observability of infinite-dimensional systems with skew-adjoint generator. $J$. Funct. Anal., 226(1):193-229, 2005.

33. K. Ramdani, T. Takahashi, and M. Tucsnak. Uniformly exponentially stable approximations for a class of second order evolution equations - Application to LQR problems. ESAIM Control Optim. Calc. Var., 13(3):503-527, 2007.

34. K. Ramdani, M. Tucsnak, and G. Weiss. Recovering the initial state of an infinitedimensional system using observers. Automatica, 46(10):1616-1625, 2010.

35. P.-A. Raviart and J.-M. Thomas. Introduction à l'Analyse Numérique des Equations aux Dérivées Partielles. Collection Mathématiques Appliquées pour la Maîtrise. [Collection of Applied Mathematics for the Master's Degree]. Masson, Paris, 1983.

36. G. Strang and G.J. Fix. An Analysis of the Finite Element Method. Prentice-Hall Inc., Englewood Cliffs, N. J., 1973. Prentice-Hall Series in Automatic Computation.

37. L.R. Tcheugoué Tébou and E. Zuazua. Uniform exponential long time decay for the space semi-discretization of a locally damped wave equation via an artificial numerical viscosity. Numer. Math., 95(3):563-598, 2003.

38. M. Tucsnak and G. Weiss. Observation and Control for Operator Semigroups. Birkhäuser Advanced Texts: Basler Lehrbücher. [Birkhäuser Advanced Texts: Basel Textbooks]. Birkhäuser Verlag, Basel, 2009.

39. C.Z. Xu, J. Deguenon, and G. Sallet. Infinite dimensional observers for vibrating systems. In Proceedings of 45th IEEE Conference on Decision and Control, pages 3979-3983, 2006. 